\title{
EXCHANGEABLE CHOICE FUNCTIONS
}

\author{
ARTHUR VAN CAMP AND GERT DE COOMAN
}

\begin{abstract}
We investigate how to model exchangeability with choice functions. Exchangeability is a structural assessment on a sequence of uncertain variables. We show how such assessments constitute a special kind of indifference assessment, and how this idea leads to a counterpart of de Finetti's Representation Theorem, both in a finite and a countable context.
\end{abstract}

\section{INTRODUCTION}

In this paper, we study how to model exchangeability, a structural assessment for uncertainty models that is important for inference purposes, in the framework of choice functions, an interesting approach to modelling uncertainty. This work builds on earlier results by De Cooman and Quaeghebeur [6], De Cooman et al. [7] for sets of desirable gambles.

Choice functions are related to the fundamental problem in decision theory: how to make a choice from within a set of available options. In their book, von Neumann and Morgenstern [27] provide an axiomatisation of choice based on pairwise comparisons between options. Later on, many authors [2, 15, 22] generalised this idea and proposed a theory of choice functions based on choice between more than two elements. One of the aspects of Rubin's [15] theory is that, between any pair of options, the agent either prefers one of them or is indifferent between them, so two options are never incomparable. However, the agent may be undecided between two options without being indifferent between them; this will for instance typically be the case when there is no relevant information available. This is one of the motivations for a theory of imprecise probabilities [28], where incomparability and indifference are distinguished. Kadane et al. [12] and Seidenfeld et al. [19] generalise Rubin's [15] axioms to allow for incomparability.

Exchangeability is a structural assessment on a sequence of uncertain variables. Loosely speaking, making a judgement of exchangeability means that the order in which the variables are observed is considered irrelevant. This irrelevancy will be modelled through an indifference assessment. The first detailed study of exchangeability was given by de Finetti [8]; see Reference [9] for an overview of finite exchangeability for classical probability theory. We refer to the paper by De Cooman and Quaeghebeur [6, Section 1] for a brief historical overview.

In Section 2, we recall the necessary tools for modelling indifference with choice functions. Next, in Section 4, we derive de Finetti-like Representation Theorems for a finite sequence that is exchangeable. We take this one step further in Section 5, where we consider a countable sequence and derive a representation theorem for such sequences. In order to allow comparison with earlier work [6], we also provide representation theorems for sets of desirable gambles. 


\section{Choice FunCtions, Desirability AND INDIFFERENCE}

Consider a real vector space $\mathcal{V}$, provided with the vector addition and scalar multiplication. Elements $u$ of $\mathcal{V}$ are intended as abstract representations of options amongst which a subject can express his preferences, by specifying, as we will see below, choice functions. Often, options are bounded real-valued maps on the possibility space, interpreted as uncertain rewards, and therefore also called gambles. The set of all gambles on some domain $\mathcal{X}$ will be denoted by $\mathcal{L}(\mathcal{X})$. In this paper, we will rather focus on vector-valued gambles, because earlier work by Zaffalon and Miranda [30] has already shown that this leads to an approach to modelling uncertainty that is even more general than the typical imprecise probability approach. Moreover, as we have discussed in some detail in [26, Section 3], the account of coherent choice functions that Seidenfeld et al. [19] consider, can be embedded into our framework, under some mild conditions. To focus the ideas, let $\mathcal{X}$ be an arbitrary possibility space, and $\mathcal{R}$ be a finite set ${ }^{1}$ With a vector-valued gamble $f$ on $\mathcal{X}$ we mean an element of the set $\mathcal{L}(\mathcal{X} \times \mathcal{R})$ of gambles on the domain $\mathcal{X} \times \mathcal{R}$ : indeed, for every $x$ in $\mathcal{X}$, the partial map $f(x, \bullet)$ is a vector in $\mathbb{R}^{|\mathcal{R}|}$. We will commonly refer to $\mathcal{X}$ as the 'state part' of the domain, and to $\mathcal{R}$ as the 'rewards part'. Of course, by letting $|\mathcal{R}|=1$, we retrieve the set $\mathcal{L}(\mathcal{X})$ of (real-valued) gambles.

However, we will define choice functions on general real vector spaces $\mathcal{V}$ rather than on the more specific $\mathcal{L}(\mathcal{X} \times \mathcal{R})$, because, as we will see later, we will need to define choice functions on equivalence classes of gambles, which are no longer gambles themselves, but still constitute a vector space ${ }^{2}$ Given any subset $A$ of $\mathcal{V}$, we will define the linear hull

$$
\operatorname{span}(A):=\left\{\sum_{k=1}^{n} \lambda_{k} u_{k}: n \in \mathbb{N}, \lambda_{k} \in \mathbb{R}, u_{k} \in A\right\} \subseteq \mathcal{V}
$$

and the positive hull

$$
\operatorname{posi}(A):=\left\{\sum_{k=1}^{n} \lambda_{k} u_{k}: n \in \mathbb{N}, \lambda_{k} \in \mathbb{R}_{>0}, u_{k} \in A\right\} \subseteq \operatorname{span}(A),
$$

where $\mathbb{R}_{>0}$ is the set of all (strictly) positive real numbers. Furthermore, for any $\lambda$ in $\mathbb{R}_{>0}$ and $u$ in $\mathcal{V}$, we let $\lambda A+\{v\}:=\{\lambda u+v: u \in A\}$. A subset $A$ of $\mathcal{V}$ is called a convex cone if it is closed under positive finite linear combinations, i.e. if $\operatorname{posi}(A)=A$. A convex cone $\mathcal{K}$ is called proper if $\mathcal{K} \cap-\mathcal{K}=\{0\}$. With any proper convex cone $\mathcal{K} \subseteq \mathcal{V}$, we associate a vector ordering $\leq_{\mathcal{K}}$ on $\mathcal{V}$ as follows: $u \leq_{\mathcal{K}} v \Leftrightarrow v-u \in \mathcal{K}$ for any $u$ and $v$ in $\mathcal{V}$. For any $u$ and $v$ in $\mathcal{V}$, we write $u<_{\mathcal{K}} v$ if $u \leq_{\mathcal{K}} v$ and $u \neq v$. We collect all the options $u$ for which $0<_{\mathcal{K}} u$ in $\mathcal{V}_{>0}$. When we work with vector-valued gambles, then $\mathcal{V}=\mathcal{L}(\mathcal{X} \times \mathcal{R})$ and the ordering will be the standard one $\leq$, given by

$$
f \leq g \Leftrightarrow(\forall x \in \mathcal{X}, r \in \mathcal{R}) f(x, r) \leq g(x, r) \Leftrightarrow(\forall x \in \mathcal{X}) f(x, \bullet) \leq g(x, \bullet)
$$

We collect the positive gambles_-gambles $f$ for which $0<f$-in $\mathcal{L}(\mathcal{X} \times \mathcal{R})_{>0}$. Then $\leq$ corresponds to $\leq_{\mathcal{K}}$ where we let $\mathcal{K}:=\mathcal{L}(\mathcal{X} \times \mathcal{R})_{>0} \cup\{0\}$.

We denote by $\mathcal{Q}(\mathcal{V})$ the set of all non-empty finite subsets of $\mathcal{V}$. Elements of $\mathcal{Q}(\mathcal{V})$ are the option sets amongst which a subject can choose his preferred options.

\footnotetext{
${ }^{1}$ Mostly, $\mathcal{R}$ is interpreted as a set of 'rewards', but it need not have an interpretation. We can allow for countable $\mathcal{R}$ provided we then restrict ourselves to the linear space of those gambles $f$ on $\mathcal{X} \times \mathcal{R}$ for which $\sum_{r \in \mathcal{R}} f(\bullet, r)$ is real-valued and bounded - a gamble on $\mathcal{X}$.

${ }^{2}$ This also allows us to connect our approach with the theory of coherent choice functions by Seidenfeld et al. [19], where the authors define their choice function on horse lotteries instead of gambles.
} 
A choice function $C$ on $\mathcal{V}$ is a map $C: \mathcal{Q} \rightarrow \mathcal{Q} \cup\{\varnothing\}: A \mapsto C(A)$ such that $C(A) \subseteq A$. The idea underlying this definition is that a choice function $C$ selects the set $C(A)$ of 'best' options in the option set $A$, or, on another interpretation, the ones that cannot be rejected. Our definition resembles the one commonly used in the literature [1, 19, 21], except for a restriction to finite option sets $]^{3}$ which, then again, is also not altogether unusual [10, 16, 20].

Not every such map represents rational beliefs; only the coherent ones are considered to do so.

Definition 1 (Coherent choice function). We call a choice function $C$ on $\mathcal{V}$ coherent ${ }^{4}$ if for all $A, A_{1}$ and $A_{2}$ in $\mathcal{Q}(\mathcal{V}), u$ and $v$ in $\mathcal{V}$, and $\lambda$ in $\mathbb{R}_{>0}$ :

C1. $C(A) \neq \varnothing$;

C2. if $u<v$ then $u \notin C(\{u, v\})$;

C3. a. if $C\left(A_{2}\right) \subseteq A_{2} \backslash A_{1}$ and $A_{1} \subseteq A_{2} \subseteq A$ then $C(A) \subseteq A \backslash A_{1}$;

b. if $C\left(A_{2}\right) \subseteq A_{2} \backslash A_{1}$ and $A \subseteq A_{1}$ then $C\left(A_{2} \backslash A\right) \subseteq A_{2} \backslash A_{1}$;

C4. a. if $A_{1} \subseteq C\left(A_{2}\right)$ then $\lambda A_{1} \subseteq C\left(\lambda A_{2}\right)$;

b. if $A_{1} \subseteq C\left(A_{2}\right)$ then $A_{1}+\{u\} \subseteq C\left(A_{2}+\{u\}\right)$.

It will sometimes be easier to use rejection functions instead of choice functions. A rejection function $R$ identifies from within every option set $A$, the options that are rejected, or not chosen: $R(A):=A \backslash C(A)$. Formally, a rejection function $R$ on $\mathcal{V}$ is a map $R: \mathcal{Q} \rightarrow$ $\mathcal{Q} \cup\{\varnothing\}: A \mapsto R(A)$ such that $R(A) \subseteq A$. We can equivalently express the rationality axioms in terms of rejection functions:

Definition 2 (Coherent rejection function). We call a rejection function $R$ on $\mathcal{V}$ coherent if for all $A, A_{1}$ and $A_{2}$ in $\mathcal{Q}$, all $u$ and $v$ in $\mathcal{V}$, and all $\lambda$ in $\mathbb{R}_{>0}$ :

R1. $R(A) \neq A$;

R2. if $u<v$ then $u \in R(\{u, v\})$;

R3. a. if $A_{1} \subseteq R\left(A_{2}\right)$ and $A_{2} \subseteq A$ then $A_{1} \subseteq R(A)$;

b. if $A_{1} \subseteq R\left(A_{2}\right)$ and $A \subseteq A_{1}$ then $A_{1} \backslash A \subseteq R\left(A_{2} \backslash A\right)$;

R4. a. if $A_{1} \subseteq R\left(A_{2}\right)$ then $\lambda A_{1} \subseteq R\left(\lambda A_{2}\right)$;

b. if $A_{1} \subseteq R\left(A_{2}\right)$ then $A_{1}+\{u\} \subseteq R\left(A_{2}+\{u\}\right)$.

Consider two isomorphic vector spaces $\mathcal{V}$ and $\mathcal{W}$, a linear order isomorphism $\phi$ between $\mathcal{V}$ and $\mathcal{W}$, and a choice function $C$ on $\mathcal{V}$. Define the choice function $C^{\prime}$ on $\mathcal{W}$ as $u \in C(A) \Leftrightarrow$ $\phi(u) \in C^{\prime}(\phi(A))$ for all $A$ in $\mathcal{Q}(\mathcal{V})$ and $u$ in $A$. Then, because $\phi$ is a bijection, $C$ satisfies Axioms $\mathrm{C} 1$ and $\mathrm{C} 3$ if and only if $C^{\prime}$ does; furthermore, because $\phi$ is order preserving, $C$ satisfies Axiom $C 2$ if and only if $C^{\prime}$ does; and finally, because $\phi$ is linear, $C$ satisfies Axiom $\mathrm{C} 4$ if and only if $C^{\prime}$ does: such isomorphisms preserve coherence.

With any rejection function $R$, we let correspond a set of desirable options (or gambles) $D_{R}=\{u \in \mathcal{V}: 0 \in R(\{0, u\})\}$. We can rewrite this in terms of the corresponding choice function $C$, resulting in the same set of desirable options $D_{C}:=\{u \in \mathcal{V}: 0 \notin C(\{0, u\})\}=D_{R}$. A set of desirable options need not be derived from a choice function or a rejection function.

\footnotetext{
${ }^{3}$ The reason for our restriction to finite option sets is mainly a technical one: for instance the proof of Proposition 19 relies on the finiteness of the option sets. We refer to [23] for more information about this.

${ }^{4}$ Our rationality axioms are based on those by Seidenfeld et al. [19], slightly modified for use with sets of desirable options. Seidenfeld et al. [19] use horse lotteries as options, but, as mentioned before, by using vector-valued gambles their account of coherent choice functions can be embedded into ours; see our earlier work [26]. We would like to note here that Seidenfeld et al. [19] have a different definition of coherence: they call a choice function coherent if it is represented through E-admissibility by some set of (finitely-additive) real-valued probabilities and real-valued cardinal utilities. They introduce 4 axioms on choice functions that are equivalent (Seidenfeld et al. [19] Theorems 3 and 4]) to coherence, under some mild conditions.
} 
It is the collection of options that the agent strictly prefers to the status quo represented by the null vector 0; for relevant literature about sets of desirable options (or gambles), we refer to References [14, 18, 28, 29].

Definition 3 (Coherent set of desirable options). We call a set of desirable options $D \subseteq \mathcal{V}$ coherent if for all $u$ and $v$ in $\mathcal{V}$ and $\lambda$ in $\mathbb{R}_{>0}$ :

D1. $0 \notin D$;

D2. if $0<u$ then $u \in D$;

D3. if $u \in D$ then $\lambda u \in D$;

D4. if $u, v \in D$ then $u+v \in D$.

We can regard a set of desirable options essentially as the restriction to pairwise comparisons of a choice function. Moreover, this restriction $D_{C}$ is coherent if the choice function $C$ it is based on, is coherent.

Given a coherent set of desirable options $D$, there may be multiple coherent choice functions $C$ such that $D_{C}=D_{[}^{5}$ As shown by Bradley [3] , and Van Camp et al. [25], there is one unique least informative 6 one, which we call $C_{D}$, given by

$$
C_{D}(A):=\{u \in A:(\forall v \in A) v-u \notin D\} \text { for all } A \text { in } \mathcal{Q} .
$$

Since, as we will see, an exchangeability assessment amounts to a specific indifference assessment, we now recall how to model such assessments [25, Section 5]. Next to $D$-the options that the agent strictly prefers to 0 -we consider the options in $I \subseteq \mathcal{V}$, which the agent considers to be equivalent to the zero option.

Definition 4 (Coherent set of indifferent options). We call a set of indifferent options $I$ coherent if for all $u$ and $v$ in $\mathcal{V}$ and all $\lambda$ in $\mathbb{R}$ :

I1. $0 \in I$;

I2. if $u \in \mathcal{V}_{>0} \cup \mathcal{V}_{<0}$ then $u \notin I$;

I3. if $u \in I$ then $\lambda u \in I$;

I4. if $u, v \in I$ then $u+v \in I$.

We collect all options that are indifferent to an option $u$ in $\mathcal{V}$ into the equivalence class $[u]:=\{v \in \mathcal{V}: v-u \in I\}=\{u\}+I$. The set of all these equivalence classes is the quotient space $\mathcal{V} / I:=\{[u]: u \in \mathcal{V}\}$, a linear space itself. We provide it with the natural ordering inherited from $\mathcal{V}$ :

$$
\tilde{u} \leq \tilde{v} \Leftrightarrow(\exists u \in \tilde{u}, v \in \tilde{v}) u \leq v
$$

for all $\tilde{u}$ and $\tilde{v}$ in $\mathcal{V} / I$.

In the remainder of this section, we will recall some of the results about indifference by Van Camp et al. [25], needed for this paper. Consider any coherent set of indifferent options $I$.

Definition 5 (Compatibility of with a set of indifferent options). A choice function $C$ is called compatible with $I$ if there is some representing choice function $C^{\prime}$ on $\mathcal{V} / I$ such that

$$
C(A)=\left\{u \in A:[u] \in C^{\prime}(A / I)\right\}
$$

for all $A$ in $\mathcal{Q}(\mathcal{V})$.

\footnotetext{
${ }^{5}$ There may be even more incoherent choice functions $C$ such that $D_{C}=D$, but we are not interested in those choice functions.

${ }^{6}$ This is the minimal element of the partially ordered set of all the coherent choice functions under the partial order $\sqsubseteq$ given by $C_{1} \sqsubseteq C_{2} \Leftrightarrow(\forall A \in \mathcal{Q}) C_{1}(A) \supseteq C_{2}(A)$, for all choice functions $C_{1}$ and $C_{2}$.
} 
In this case, $C^{\prime}$ is uniquely determined by $C^{\prime}(A / I)=C(A) / I$ for all $A$ in $\mathcal{Q}(\mathcal{V})$, and, moreover, $C$ is coherent if and only if $C^{\prime}$ is. Equivalently, we have the following useful characterisation: $C$ is compatible with $I$ if and only if

$$
0 \in C(A) \Leftrightarrow u \in C(A) \text {, for all } u \text { in } I \text { and } A \supseteq\{0, u\} \text { in } \mathcal{Q}(\mathcal{V}),
$$

which corresponds to the definition of indifference given by Seidenfeld [17].

For desirability, compatibility with a coherent set of indifferent options $I$ is defined as follows. We call a set of desirable gambles $D$ compatible with $I$ if $D+I \subseteq D$, and this is equivalent to $D=\cup D^{\prime}$ where $D^{\prime} \subseteq \mathcal{V} / I$ is the representing set of desirable options. In that case, $D^{\prime}$ is uniquely given by $D^{\prime}=D / I$ - so $D=\bigcup_{u \in D}[u]$ —and, moreover, $D$ is coherent if and only if $D^{\prime}$ is.

\section{CONDITIONING CHOICE FUNCTIONS}

Consider a choice function $C$ on $\mathcal{L}(\mathcal{X} \times \mathcal{R})$. With a non-empty subset $B$ of $\mathcal{X}$, we associate a conditioned or updated choice function $C\rfloor B$ on $\mathcal{L}(B \times \mathcal{R})$, defined by

$$
C] B(A):=\left\{f \in A: \mathbb{I}_{B} f \in C\left(\left\{\mathbb{I}_{B} f: f \in A\right\}\right)\right\} \text { for all } A \text { in } \mathcal{Q}(\mathcal{L}(B \times \mathcal{R})) .
$$

We will also call $C\rfloor B$ a conditional choice function.

In this definition, we le $]^{7}$ for any non-empty subset $B$ of $\mathcal{X}$ and any $f$ in $\mathcal{L}(B \times \mathcal{R}), \mathbb{I}_{B} f$ be the gamble on $\mathcal{X} \times \mathcal{R}$ given by

$$
\mathbb{I}_{B} f(x, r):= \begin{cases}f(x, r) & \text { if } x \in B \\ 0 & \text { if } x \notin B\end{cases}
$$

for all $x$ in $\mathcal{X}$ and $r$ in $\mathcal{R}$. Note that, for any non-empty subset $B$ of $\mathcal{X}$, and all $f$ and $g$ in $\mathcal{L}(B \times \mathcal{R})$, we have that $\mathbb{I}_{B \times \mathcal{R}} \mathbb{I}_{B} f=\mathbb{I}_{B} f$, whence

$$
\begin{aligned}
f \neq g & \Leftrightarrow(\exists x \in B, r \in \mathcal{R})(f(x, r) \neq g(x, r)) \\
& \Leftrightarrow(\exists x \in B, r \in \mathcal{R})\left(\mathbb{I}_{B} f(x, r) \neq \mathbb{I}_{B} g(x, r)\right) \\
& \Leftrightarrow(\exists x \in \mathcal{X}, r \in \mathcal{R})\left(\mathbb{I}_{B} f(x, r) \neq \mathbb{I}_{B} g(x, r)\right) \Leftrightarrow \mathbb{I}_{B} f \neq \mathbb{I}_{B} g,
\end{aligned}
$$

and also

$$
\begin{aligned}
f<g & \Leftrightarrow(f \leq g \text { and } f \neq g) \\
& \Leftrightarrow\left((\forall x \in B, r \in \mathcal{R})(f(x, r) \leq g(x, r)) \text { and } \mathbb{I}_{B} f \neq \mathbb{I}_{B} g\right) \\
& \Leftrightarrow\left((\forall x \in B, r \in \mathcal{R})\left(\mathbb{I}_{B} f(x, r) \leq \mathbb{I}_{B} g(x, r)\right) \text { and } \mathbb{I}_{B} f \neq \mathbb{I}_{B} g\right) \\
& \Leftrightarrow\left((\forall x \in \mathcal{X}, r \in \mathcal{R})\left(\mathbb{I}_{B} f(x, r) \leq \mathbb{I}_{B} g(x, r)\right) \text { and } \mathbb{I}_{B} f \neq \mathbb{I}_{B} g\right) \\
& \Leftrightarrow\left(\mathbb{I}_{B} f \leq \mathbb{I}_{B} g \text { and } \mathbb{I}_{B} f \neq \mathbb{I}_{B} g\right) \Leftrightarrow \mathbb{I}_{B} f<\mathbb{I}_{B} g .
\end{aligned}
$$

We will use the simplifying notation $\mathbb{I}_{B} A:=\left\{\mathbb{I}_{B} f: f \in A\right\} \in \mathcal{Q}(\mathcal{L}(\mathcal{X} \times \mathcal{R}))$ for any $A$ in $\mathcal{Q}(\mathcal{L}(B \times \mathcal{R}))$, where, by Equation $[2], \mathbb{I}_{B} f$ is the gamble on $\mathcal{X} \times \mathcal{R}$, that is equal to $f$ on $B \times \mathcal{R}$ and to 0 on $B^{c} \times \mathcal{R}$. Using this notational convention,

$$
C] B(A)=\left\{f \in A: \mathbb{I}_{B} f \in C\left(\mathbb{I}_{B} A\right)\right\} \text { for all } A \text { in } \mathcal{Q}(\mathcal{L}(B \times \mathcal{R})),
$$

or equivalently,

$$
f \in C] B(A) \Leftrightarrow \mathbb{I}_{B} f \in C\left(\mathbb{I}_{B} A\right), \text { for all } A \text { in } \mathcal{Q}(\mathcal{L}(B \times \mathcal{R})) \text { and all } f \text { in } A .
$$

The following property, which guarantees that conditioning preserves coherence, has been established by Van Camp [23, Proposition 150]:

\footnotetext{
${ }^{7} \mathbb{I}_{B}$ is the indicator (gamble) defined on $\mathcal{X}$ - that assumes the value 1 on $B$ and 0 on $B^{c}$-, while $f$ is a gamble defined on $B \times \mathcal{R}$ : their domains differ, so their multiplication needs to be defined with some care.
} 
Proposition 1. Consider any choice function $C$ on $\mathcal{L}(\mathcal{X} \times \mathcal{R})$ and any non-empty subset $B$ of $\mathcal{X}$. If $C$ is coherent, then so is $C\rfloor B$.

\section{Finite EXChangeabiLity}

Consider $n$ in $\mathbb{N}$ variables $X_{1}, \ldots, X_{n}$ taking values in a non-empty finite set $\mathcal{X}$. The finite possibility space for the uncertain sequence $X=\left(X_{1}, \ldots, X_{n}\right)$ is $\mathcal{X}^{n}$.

We denote by $x=\left(x_{1}, \ldots, x_{n}\right)$ an arbitrary element of $\mathcal{X}^{n}$. For any $n$ in $\mathbb{N}$ we let $\mathcal{P}_{n}$ be the group of all permutations of the index set $\{1, \ldots, n\}$. There are $\left|\mathcal{P}_{n}\right|=n$ ! such permutations. With any such permutation $\pi$, we associate a permutation of $\mathcal{X}^{n}$, also denoted by $\pi$, and defined by $(\pi x)_{k}:=x_{\pi(k)}$ for every $k$ in $\{1, \ldots, n\}$, or in other words, $\pi\left(x_{1}, \ldots, x_{n}\right)=$ $\left(x_{\pi(1)}, \ldots, x_{\pi(n)}\right): \pi x$ is obtained from $x$ by permuting the indices of its components. Similarly, we lift $\pi$ to a permutation $\pi^{t}$ on $\mathcal{L}\left(\mathcal{X}^{n} \times \mathcal{R}\right)$ by letting $\left(\pi^{t} f\right)(x, r):=f(\pi x, r)$ for all $x$ in $\mathcal{X}^{n}$ and $r$ in $\mathcal{R}$. Observe that $\pi^{t}$ is a linear permutation of the vector space $\mathcal{L}\left(\mathcal{X}^{n} \times \mathcal{R}\right)$ of all vector-valued gambles on $\mathcal{X}^{n}$.

If a subject assesses that the sequence of variables $X$ in $\mathcal{X}^{n}$ is exchangeable, this means that he is indifferent between any gamble $f$ in $\mathcal{L}\left(\mathcal{X}^{n} \times \mathcal{R}\right)$ and its permuted variant $\pi^{t} f$, for all $\pi$ in $\mathcal{P}_{n}$. This leads to the following set of indifferent gambles:

$$
I_{\mathcal{P}_{n}}:=\operatorname{span}\left\{f-\pi^{t} f: f \in \mathcal{L}\left(\mathcal{X}^{n} \times \mathcal{R}\right) \text { and } \pi \in \mathcal{P}_{n}\right\} .
$$

Definition 6 (Finite exchangeability). A choice function $C$ on $\mathcal{L}\left(\mathcal{X}^{n} \times \mathcal{R}\right)$ is called (finitely) exchangeable if it is compatible with $I_{\mathcal{P}_{n}}$. Similarly, a set of desirable gambles $D \subseteq \mathcal{L}\left(\mathcal{X}^{n} \times\right.$ $\mathcal{R}$ ) is called (finitely) exchangeable if it is compatible with $I_{\mathcal{P}_{n}}$.

Of course, so far, we do not yet know whether this notion of exchangeability is well-defined: indeed, we do not know yet whether $I_{\mathcal{P}_{n}}$ is a coherent set of indifferent gambles, in the sense of Definition 4 In the next section, we will show that this is indeed the case. But once we have established that $I_{\mathcal{P}_{n}}$ is a coherent set of indifferent gambles, exchangeability is nothing more fancy than compatibility with $I_{\mathcal{P}_{n}}$. What we will do below, is use this general representation result to obtain a particular equivalent representation result for exchangeable choice functions, in terms of (vector-valued) gambles on count vectors.

4.1. Count vectors. Let us now provide the tools necessary to prove that $I_{\mathcal{P}_{n}}$ is a coherent set of indifferent gambles, as introduced in Definition 4

The permutation invariant atoms $[x]:=\left\{\pi x: x \in \mathcal{X}^{n}\right\}$, with $x$ in $\mathcal{X}^{n}$, are the smallest permutation invariant subsets of $\mathcal{X}^{n}$. We consider the counting map

$$
T: \mathcal{X}^{n} \rightarrow \mathcal{N}^{n}: x \mapsto T(x)
$$

where $T(x)$ is called the count vector of $x$. It is the $\mathcal{X}$-tuple with components $T_{z}(x):=$ $\left|\left\{k \in\{1, \ldots, n\}: x_{k}=z\right\}\right|$ for all $z$ in $\mathcal{X}$, so $T_{z}(x)$ is the number of times that $z$ occurs in the sequence $x_{1}, \ldots, x_{n}$. The range of $T$ - the set $\mathcal{N}^{n}$-is called the set of possible count vectors and is given by

$$
\mathcal{N}^{n}:=\left\{m \in \mathbb{Z}_{\geq 0}^{\mathcal{X}}: \sum_{x \in \mathcal{X}} m_{x}=n\right\} .
$$

Applying any permutation to $x$ leaves its result under the counting map unchanged:

$$
T(x)=T(\pi x) \text { for al } x \text { in } \mathcal{X}^{n} \text { and } \pi \text { in } \mathcal{P}_{n} .
$$

For any $x$ in $\mathcal{X}^{n}$, if $m=T(x)$ then $[x]=\left\{y \in \mathcal{X}^{n}: T(y)=m\right\}$, so the permutation invariant atom $[x]$ is completely determined by the count vector $m$ of all its elements, and is therefore 
also denoted by $[T(x)]=[m]$. Remark that $\left\{[m]: m \in \mathcal{N}^{n}\right\}$ partitions $\mathcal{X}^{n}$ into disjoint parts with constant count vectors, and that $|[m]|=\left(\begin{array}{c}n \\ m\end{array}\right):=\frac{n !}{\prod_{z \in \mathcal{X}} m_{z} !}$.

In order to extend the application of the count vectors for use with gambles, let us define the set of all permutation invariant vector-valued gambles as

$$
\mathcal{L}_{\mathcal{P}_{n}}\left(\mathcal{X}^{n} \times \mathcal{R}\right):=\left\{f \in \mathcal{L}\left(\mathcal{X}^{n} \times \mathcal{R}\right):\left(\forall \pi \in \mathcal{P}_{n}\right) \pi^{t} f=f\right\} \subseteq \mathcal{L}\left(\mathcal{X}^{n} \times \mathcal{R}\right),
$$

and a special transformation $\operatorname{inv}_{\mathcal{P}_{n}}$ of the linear space $\mathcal{L}\left(\mathcal{X}^{n} \times \mathcal{R}\right)$ given by

$$
\operatorname{inv}_{\mathcal{P}_{n}}: \mathcal{L}\left(\mathcal{X}^{n} \times \mathcal{R}\right) \rightarrow \mathcal{L}\left(\mathcal{X}^{n} \times \mathcal{R}\right): f \mapsto \operatorname{inv}_{\mathcal{P}_{n}}(f):=\frac{1}{n !} \sum_{\pi \in \mathcal{P}_{n}} \pi^{t} f
$$

which, as we will see in the following proposition, is closely linked with $\mathcal{L}_{\mathcal{P}_{n}}\left(\mathcal{X}^{n} \times \mathcal{R}\right.$ ) (see also References [6, 25] for a proof).

Proposition 2. $\operatorname{inv}_{\mathcal{P}_{n}}$ is a linear transformation of $\mathcal{L}\left(\mathcal{X}^{n} \times \mathcal{R}\right)$, and

(i) $\operatorname{inv}_{\mathcal{P}_{n}} \circ \pi^{t}=\operatorname{inv}_{\mathcal{P}_{n}}=\pi^{t} \circ \operatorname{inv}_{\mathcal{P}_{n}}$ for all $\pi$ in $\mathcal{P}$;

(ii) $\operatorname{inv}_{\mathcal{P}_{n}} \circ \operatorname{inv}_{\mathcal{P}_{n}}=\operatorname{inv}_{\mathcal{P}_{n}}$;

(iii) $\operatorname{ker}\left(\operatorname{inv}_{\mathcal{P}_{n}}\right)=I_{\mathcal{P}_{n}}$;

(iv) $\operatorname{rng}\left(\operatorname{inv}_{\mathcal{P}_{n}}\right)=\mathcal{L}_{\mathcal{P}_{n}}\left(\mathcal{X}^{n} \times \mathcal{R}\right)$.

Moreover, for any $f$ and $g$ in $\mathcal{L}\left(\mathcal{X}^{n} \times \mathcal{R}\right)$, we have that $g \in f / I_{\mathcal{P}_{n}} \Leftrightarrow \operatorname{inv}_{\mathcal{P}_{n}} g=\operatorname{inv}_{\mathcal{P}_{n}} f$.

So we see that $\operatorname{inv}_{\mathcal{P}_{n}}$ is a linear projection operator that renders a vector-valued gamble insensitive to permutation (or permutation invariant) by replacing it with the uniform average of all its permutations. As a result, it assumes the same value for all vector-valued gambles that can be related to each other through some permutation: $\operatorname{inv}_{\mathcal{P}_{n}}(f)=\operatorname{inv}_{\mathcal{P}_{n}}(g)$ if $f=\pi^{t} g$ for some $\pi$ in $\mathcal{P}_{n}$, for all $f$ and $g$ in $\mathcal{L}\left(\mathcal{X}^{n} \times \mathcal{R}\right)$. Furthermore, for any $f$ in $\mathcal{L}\left(\mathcal{X}^{n} \times \mathcal{R}\right)$, its transformation $\operatorname{inv}_{\mathcal{P}_{n}}(f)$ is permutation invariant and therefore constant on the permutation invariant atoms $[m]:\left(\operatorname{inv}_{\mathcal{P}_{n}}(f)\right)(x, r)=\left(\operatorname{inv}_{\mathcal{P}_{n}}(f)\right)(y, r)$ if $[x]=[y]$, for all $x$ and $y$ in $\mathcal{X}^{n}$, and $r$ in $\mathcal{R}$. We can use the properties of $\operatorname{inv}_{\mathcal{P}_{n}}$ to prove that $I_{\mathcal{P}_{n}}$ is coherent and therefore well suited for our definition of exchangeability.

Proposition 3. For any $n$ in $\mathbb{N}$, the set $I_{\mathcal{P}_{n}}$, defined in Equation (3), is a coherent set of indifferent vector-valued gambles.

Proof. For Axiom [1], since $I_{\mathcal{P}_{n}}$ is a linear hull by its Definition [3], 0 is included in $I_{\mathcal{P}_{n}}$. For Axiom 12, consider any $f$ in $I_{\mathcal{P}_{n}}$ and assume ex absurdo that $f \in \mathcal{L}\left(\mathcal{X}^{n} \times \mathcal{R}\right)_{>0} \cup$ $\mathcal{L}\left(\mathcal{X}^{n} \times \mathcal{R}\right)<0$. If $f \in \mathcal{L}\left(\mathcal{X}^{n} \times \mathcal{R}\right)_{>0}$ then $\pi^{t} f \in \mathcal{L}\left(\mathcal{X}^{n} \times \mathcal{R}\right)_{>0}$ for all $\pi$ in $\mathcal{P}_{n}$, and therefore $\operatorname{inv}_{\mathcal{P}_{n}}(f)>0$, a contradiction with Proposition 2(iii) If $f \in \mathcal{L}\left(\mathcal{X}^{n} \times \mathcal{R}\right)_{<0}$ then, similarly $\operatorname{inv}_{\mathcal{P}_{n}}(f)<0$, again a contradiction with Proposition [2(iii). Axioms 13 and I4 are satisfied because $I_{\mathcal{P}_{n}}$ is a linear hull.

Since $I_{\mathcal{P}_{n}}$ is coherent, exchangeability is well-defined: a choice function $C_{n}$ on $\mathcal{L}\left(\mathcal{X}^{n} \times\right.$ $\mathcal{R})$ and a set of desirable vector-valued gambles $D_{n} \subseteq \mathcal{L}\left(\mathcal{X}^{n} \times \mathcal{R}\right)$ are exchangeable if they are compatible with the coherent set of indifferent vector-valued gambles $I_{\mathcal{P}_{n}}$. By Definition 5. $C_{n}$ is therefore represented by a choice function $C^{\prime}$ on $\mathcal{L}\left(\mathcal{X}^{n} \times \mathcal{R}\right) / I_{\mathcal{P}_{n}}$, and similarly, $D_{n}$ is represented by a set of desirable gambles $D^{\prime} \subseteq \mathcal{L}\left(\mathcal{X}^{n} \times \mathcal{R}\right) / I_{\mathcal{P}_{n}}$. So we can focus on the quotient space and its elements: equivalence classes of mutually indifferent vector-valued gambles.

But before we do that in the next section, it will pay to further explore the notions we have introduced thus far. Consider any $f$ in $\mathcal{L}\left(\mathcal{X}^{n} \times \mathcal{R}\right)$. What is the constant value that $\operatorname{inv}_{\mathcal{P}_{n}}(f)$ assumes on a permutation invariant atom $[m]$ ? To answer this question, consider 
any $x$ in $[m]$, then

$$
\begin{aligned}
\left(\operatorname{inv}_{\mathcal{P}_{n}}(f)\right)(x, \bullet)=\frac{1}{n !} \sum_{\pi \in \mathcal{P}_{n}} f(\pi x, \bullet) & =\frac{1}{n !} \frac{\left|\mathcal{P}_{n}\right|}{|[m]|} \sum_{y \in\left\{\pi x: \pi \in \mathcal{P}_{n}\right\}} f(y, \bullet) \\
& =\frac{1}{\left(\begin{array}{c}
n \\
m
\end{array}\right)} \sum_{y \in[x]} f(y, \bullet)=\frac{1}{\left(\begin{array}{c}
n \\
m
\end{array}\right)} \sum_{y \in[m]} f(y, \bullet)
\end{aligned}
$$

where we used the fact that $\left|\mathcal{P}_{n}\right|=n$ ! and $|[m]|=\left(\begin{array}{c}n \\ m\end{array}\right)$. Therefore, for all $r$ in $\mathcal{R}$,

$$
\left.\operatorname{(inv}_{\mathcal{P}_{n}}(f)\right)(\bullet, r)=\sum_{m \in \mathcal{N}^{n}} \mathrm{H}_{n}(f(\bullet, r) \mid m) \mathbb{I}_{[m]},
$$

where $\mathrm{H}_{n}(\bullet \mid m)$ is the linear expectation operator associated with the uniform distribution on the invariant atom $[m]$ :

$$
\mathrm{H}_{n}(g \mid m):=\frac{1}{\left(\begin{array}{l}
n \\
m
\end{array}\right)} \sum_{y \in[m]} g(y) \text { for all } g \text { in } \mathcal{L}\left(\mathcal{X}^{n}\right) \text { and } m \text { in } \mathcal{N}^{n} .
$$

It characterises a (multivariate) hyper-geometric distribution [11], associated with random sampling without replacement from an urn with $n$ balls of types $\mathcal{X}$, whose composition is characterised by the count vector $m$.

The result of subjecting a gamble $f$ on $\mathcal{X}^{n} \times \mathcal{R}$ to the map

$$
\begin{aligned}
\mathrm{H}_{n}: \mathcal{L}\left(\mathcal{X}^{n} \times \mathcal{R}\right) \rightarrow & \mathcal{L}\left(\mathcal{N}^{n} \times \mathcal{R}\right): f \mapsto \mathrm{H}_{n}(f) \\
& \text { with }\left(\mathrm{H}_{n}(f)\right)(m, r):=\mathrm{H}_{n}(f(\bullet, r) \mid m) \text { for all } m \text { in } \mathcal{N} \text { and } r \text { in } \mathcal{R},
\end{aligned}
$$

is the gamble $\mathrm{H}_{n}(f)$ on $\mathcal{N}^{n} \times \mathcal{R}$ that assumes the value $\frac{1}{\left(\begin{array}{c}n \\ m\end{array}\right)} \sum_{y \in[m]} f(y, r)$ in every $m$ in $\mathcal{N}^{n}$ and $r$ in $\mathcal{R}$.

4.2. Exchangeable equivalence classes of gambles. We already know that exchangeable choice functions are represented by choice functions on the quotient space $\mathcal{L}\left(\mathcal{X}^{n} \times \mathcal{R}\right) / I_{\mathcal{P}_{n}}$, and similarly for sets of desirable gambles. In the quest for an elegant representation theorem, we thus need to focus on the quotient space $\mathcal{L}\left(\mathcal{X}^{n} \times \mathcal{R}\right) / I_{\mathcal{P}_{n}}$ and its elements, which are 'exchangeable' equivalence classes of vector-valued gambles.

In this section we investigate how the representation of permutation invariant gambles helps us find a representation result for exchangeable choice functions. This representation will use equivalence classes $[f]:=f / I_{\mathcal{P}_{n}}=\{f\}+I_{\mathcal{P}_{n}}$ of gambles, for any $f$ in $\mathcal{L}\left(\mathcal{X}^{n} \times \mathcal{R}\right)$. Recall that the quotient space $\mathcal{L}\left(\mathcal{X}^{n} \times \mathcal{R}\right) / I_{\mathcal{P}_{n}}:=\left\{[f]: f \in \mathcal{L}\left(\mathcal{X}^{n} \times \mathcal{R}\right)\right\}$ is a linear space itself, with additive identity $[0]=I_{\mathcal{P}_{n}}$, and therefore any element $\tilde{f}$ of $\mathcal{L}\left(\mathcal{X}^{n} \times \mathcal{R}\right) / I_{\mathcal{P}_{n}}$ is invariant under addition of $I_{\mathcal{P}_{n}}: \tilde{f}+I_{\mathcal{P}_{n}}=\tilde{f}$. Elements of $\mathcal{L}\left(\mathcal{X}^{n} \times \mathcal{R}\right) / I_{\mathcal{P}_{n}}$ will be generically denoted by $\tilde{f}$ or $\tilde{g}$.

Proposition 4. Consider any $f$ and $g$ in $\mathcal{L}\left(\mathcal{X}^{n} \times \mathcal{R}\right)$. Then $[f]=[g]$ if and only if $\mathrm{H}_{n}(f)=$ $\mathrm{H}_{n}(g)$.

Proof. By Proposition 2 we have that $[f]=[g] \Leftrightarrow \operatorname{inv}_{\mathcal{P}_{n}} f=\operatorname{inv}_{\mathcal{P}_{n}} g$, so it suffices to show that $\operatorname{inv}_{\mathcal{P}_{n}} f=\operatorname{inv}_{\mathcal{P}_{n}} g \Leftrightarrow \mathrm{H}_{n}(f)=\mathrm{H}_{n}(g)$. Observe that

$$
\begin{aligned}
\operatorname{inv}_{\mathcal{P}_{n}} f=\operatorname{inv}_{\mathcal{P}_{n}} g & \Leftrightarrow(\forall r \in \mathcal{R}) \operatorname{inv}_{\mathcal{P}_{n}}(f)(\bullet, r)=\operatorname{inv}_{\mathcal{P}_{n}}(g)(\bullet, r) \\
& \Leftrightarrow(\forall r \in \mathcal{R}) \sum_{m \in \mathcal{N}^{n}} \mathrm{H}_{n}(f(\bullet, r) \mid m) \mathbb{I}_{[m]}=\sum_{m \in \mathcal{N}^{n}} \mathrm{H}_{n}(g(\bullet, r) \mid m) \mathbb{I}_{[m]} \\
& \Leftrightarrow\left(\forall m \in \mathcal{N}^{n}, r \in \mathcal{R}\right) \mathrm{H}_{n}(f(\bullet, r) \mid m)=\mathrm{H}_{n}(g(\bullet, r) \mid m) \\
& \Leftrightarrow\left(\forall m \in \mathcal{N}^{n}, r \in \mathcal{R}\right) \mathrm{H}_{n}(f)(m, r)=\mathrm{H}_{n}(g)(m, r)
\end{aligned}
$$




$$
\Leftrightarrow \mathrm{H}_{n}(f)=\mathrm{H}_{n}(g)
$$

where the second equivalence follows from Equation (4) and the fourth one from Equation (6).

Therefore, $\mathrm{H}_{n}$ is constant on the exchangeable equivalence classes of vector-valued gambles: for any $\tilde{f}$ in $\mathcal{L}\left(\mathcal{X}^{n} \times \mathcal{R}\right) / I_{\mathcal{P}_{n}}$, we have that $\mathrm{H}_{n}(f)=\mathrm{H}_{n}(g)$ for all $f$ and $g$ in $\tilde{f}$. This means that the map $\tilde{\mathrm{H}}_{n}$, defined by:

$$
\tilde{\mathrm{H}}_{n}: \mathcal{L}\left(\mathcal{X}^{n} \times \mathcal{R}\right) / I_{\mathcal{P}_{n}} \rightarrow \mathcal{L}\left(\mathcal{N}^{n} \times \mathcal{R}\right): \tilde{f} \mapsto \mathrm{H}_{n}(f) \text { for any } f \text { in } \tilde{f} .
$$

is well defined. Proposition 4 guarantees that elements of $\mathcal{L}\left(\mathcal{X}^{n} \times \mathcal{R}\right) / I_{\mathcal{P}_{n}}$ can also be characterised using $\tilde{\mathrm{H}}_{n}$, in the sense that $\tilde{f}=\left\{f \in \mathcal{L}\left(\mathcal{X}^{n} \times \mathcal{R}\right): \mathrm{H}_{n}(f)=\tilde{\mathrm{H}}_{n}(\tilde{f})\right\}$ for all $\tilde{f}$ in $\mathcal{L}\left(\mathcal{X}^{n} \times \mathcal{R}\right) / I_{\mathcal{P}_{n}}$.

The map $\tilde{\mathrm{H}}_{n}$ takes as an argument any equivalence class of gambles, and maps it to some representing gamble on the count vectors. It will be useful later on to consider the map $\tilde{\mathrm{H}}_{n}^{-1}$ :

$$
\tilde{\mathrm{H}}_{n}^{-1}: \mathcal{L}\left(\mathcal{N}^{n} \times \mathcal{R}\right) \rightarrow \mathcal{L}\left(\mathcal{X}^{n} \times \mathcal{R}\right) / I_{\mathcal{P}_{n}}: h \mapsto\left[\sum_{m \in \mathcal{N}^{n}} h(m, \bullet) \mathbb{I}_{[m]}\right] .
$$

The notation of $\tilde{\mathrm{H}}_{n}^{-1}$ is suggestive: as it turns out, $\tilde{\mathrm{H}}_{n}$ and $\tilde{\mathrm{H}}_{n}^{-1}$ indeed are each other's inverses, and are therefore bijective.

Proposition 5. The maps $\tilde{\mathrm{H}}_{n}$ as defined in Equation (7) and $\tilde{\mathrm{H}}_{n}^{-1}$ as defined in Equation (8) are each other's inverses.

Proof. This proof is structured as follows: we show that (i) $\tilde{\mathrm{H}}_{n}^{-1} \circ \tilde{\mathrm{H}}_{n}=\mathrm{id}_{\mathcal{L}\left(\mathcal{X}^{n} \times \mathcal{R}\right) / I_{\mathcal{P}_{n}}}$, and (ii) $\tilde{\mathrm{H}}_{n} \circ \tilde{\mathrm{H}}_{n}^{-1}=\mathrm{id}_{\mathcal{L}\left(\mathcal{N}^{n} \times \mathcal{R}\right)}$, together implying that $\tilde{\mathrm{H}}_{n}$ and $\tilde{\mathrm{H}}_{n}^{-1}$ are each other's inverses.

For (i), consider any $\tilde{f}$ in $\mathcal{L}\left(\mathcal{X}^{n} \times \mathcal{R}\right) / I_{\mathcal{P}_{n}}$. We need to show that then $\tilde{\mathrm{H}}_{n}^{-1}\left(\tilde{\mathrm{H}}_{n}(\tilde{f})\right)=\tilde{f}$. Let $h$ be an arbitrary element of $\tilde{f}$, and $f:=\operatorname{inv}_{\mathcal{P}_{n}}(h)$. By Proposition 2(ii) then $\operatorname{inv}_{\mathcal{P}_{n}}(f)=$ $\operatorname{inv}_{\mathcal{P}_{n}}(h)$, so $f$ belongs to $\tilde{f}$ as well. Therefore $\tilde{\mathrm{H}}_{n}(\tilde{f})$ assumes the value $\mathrm{H}_{n}(f)(m, \bullet)=$ $\frac{1}{\left(\begin{array}{c}n \\ m\end{array}\right)} \sum_{y \in[m]} f(y, \bullet)$ on every $m$ in $\mathcal{N}^{n}$. But $f$ is constant on every permutation invariant atom $[m]$, so $\mathrm{H}_{n}(f)(m, \bullet)=\frac{1}{\left(\begin{array}{c}n \\ m\end{array}\right)}|[m]| f(x, \bullet)=f(x, \bullet)$ for every $x$ in $[m]$, and therefore

$$
f=\sum_{m \in \mathcal{N}^{n}} \mathrm{H}_{n}(f)(m, \bullet) \mathbb{I}_{[m]}=\sum_{m \in \mathcal{N}^{n}} \tilde{\mathrm{H}}_{n}(\tilde{f})(m, \bullet) \mathbb{I}_{[m]},
$$

where the second equality follows from Equation (7) and because $f \in \tilde{f}$. Then indeed $\tilde{\mathrm{H}}_{n}^{-1}\left(\tilde{\mathrm{H}}_{n}(\tilde{f})\right)=\left[\sum_{m \in \mathcal{N}^{n}} \tilde{\mathrm{H}}_{n}(\tilde{f})(m, \bullet) \mathbb{I}_{[m]}\right]=[f]=\tilde{f}$, where the first equality follows from Equation (8), the second one from Equation (9), and the last one from the fact that $f \in \tilde{f}$ and therefore $[f]=\tilde{f}$.

For (ii), consider any $h$ in $\mathcal{L}\left(\mathcal{N}^{n} \times \mathcal{R}\right)$. We need to show that then $\tilde{\mathrm{H}}_{n}\left(\tilde{\mathrm{H}}_{n}^{-1}(h)\right)=h$. Let $f:=\sum_{m \in \mathcal{N}^{n}} h(m, \bullet) \mathbb{I}_{[m]}$, a gamble on $\mathcal{X}^{n} \times \mathcal{R}$. Then $\tilde{\mathrm{H}}_{n}^{-1}(h)=[f]$ by Equation 8 , so $\tilde{\mathrm{H}}_{n}\left(\tilde{\mathrm{H}}_{n}^{-1}(h)\right)=\tilde{\mathrm{H}}_{n}([f])$, and since $f \in[f]$, we find using Equation (7) that $\tilde{\mathrm{H}}_{n}([f])=\mathrm{H}_{n}(f)$ and therefore $\tilde{\mathrm{H}}_{n}\left(\tilde{\mathrm{H}}_{n}^{-1}(h)\right)=\mathrm{H}_{n}(f)$. The proof is finished if we can show that $\mathrm{H}_{n}(f)=h$. Consider any $m^{\prime}$ in $\mathcal{N}^{n}$, and observe that

$$
\begin{aligned}
\mathrm{H}_{n}(f)\left(m^{\prime}, \bullet\right)=\frac{1}{\left(\begin{array}{c}
n \\
m^{\prime}
\end{array}\right)} \sum_{y \in\left[m^{\prime}\right]} f(y, \bullet) & =\frac{1}{\left(\begin{array}{c}
n \\
m^{\prime}
\end{array}\right)} \sum_{y \in\left[m^{\prime}\right]} \sum_{m \in \mathcal{N}^{n}} h(m, \bullet) \mathbb{I}_{[m]}(y) \\
& =\sum_{m \in \mathcal{N}^{n}} h(m, \bullet) \frac{1}{\left(\begin{array}{c}
n \\
m^{\prime}
\end{array}\right)} \sum_{y \in\left[m^{\prime}\right]} \mathbb{I}_{[m]}(y) \\
& =\sum_{m \in \mathcal{N}^{n}} h(m, \bullet) \mathbb{I}_{\{m\}}\left(m^{\prime}\right)=h\left(m^{\prime}, \bullet\right),
\end{aligned}
$$


where the first equality follows from Equation (5) and the penultimate one from the facts that $\left\{[m]: m \in \mathcal{N}^{n}\right\}$ partitions $\mathcal{X}^{n}$ and $\left|\left[m^{\prime}\right]\right|=\left(\begin{array}{c}n \\ m^{\prime}\end{array}\right)$, so

$$
\frac{1}{\left(\begin{array}{c}
n \\
m^{\prime}
\end{array}\right)} \sum_{y \in\left[m^{\prime}\right]} \mathbb{I}_{[m]}(y)=\left\{\begin{array}{ll}
1 & \text { if } m^{\prime}=m \\
0 & \text { otherwise }
\end{array}=\mathbb{I}_{\{m\}}\left(m^{\prime}\right) .\right.
$$

Therefore indeed $h=\mathrm{H}_{n}(f)=\tilde{\mathrm{H}}_{n}\left(\tilde{\mathrm{H}}_{n}^{-1}(h)\right)$.

The importance of Proposition 5 lies in the fact that now, $\tilde{\mathrm{H}}_{n}$ is a bijection between $\mathcal{L}\left(\mathcal{X}^{n} \times \mathcal{R}\right) / I_{\mathcal{P}_{n}}$ and $\mathcal{L}\left(\mathcal{N}^{n} \times \mathcal{R}\right)$, and therefore, exchangeable equivalence classes of gambles are in a one-to-one correspondence with gambles on count vectors.

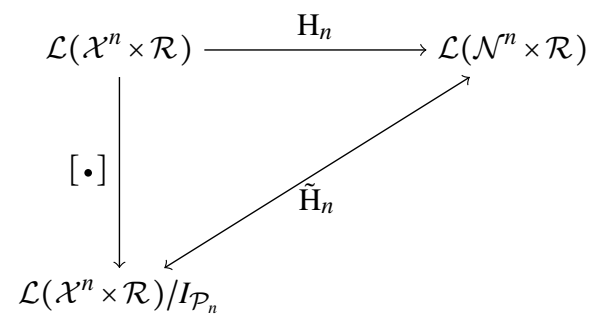

FIGURE 1. Commuting diagram for $\mathrm{H}_{n}$ and $\tilde{\mathrm{H}}_{n}$

The commuting diagram of Figure 1 above illustrates the surjections $[\bullet]: \mathcal{L}\left(\mathcal{X}^{n} \times \mathcal{R}\right) \rightarrow$ $\mathcal{L}\left(\mathcal{X}^{n} \times \mathcal{R}\right) / I_{\mathcal{P}_{n}}: f \mapsto[f]$ and $\mathrm{H}_{n}$ (indicated with a single arrow), and the bijection $\tilde{\mathrm{H}}_{n}$ (indicated with a double arrow). Since the representing choice function $C^{\prime}$ is defined from $C_{n}$ through [ $\cdot$ ] - working point-wise on sets-this already suggests that $C^{\prime}$ can be transformed into a choice function on $\mathcal{L}\left(\mathcal{N}^{n} \times \mathcal{R}\right)$. To prove that they preserve coherence, there is only one missing link: it suffices to show that the map $\tilde{\mathrm{H}}_{n}$ is linear and preserves the ordering between $\mathcal{L}\left(\mathcal{X}^{n} \times \mathcal{R}\right) / I_{\mathcal{P}_{n}}$ and $\mathcal{L}\left(\mathcal{N}^{n} \times \mathcal{R}\right)$.

Therefore, to define the ordering $\leq$ on $\mathcal{L}\left(\mathcal{X}^{n} \times \mathcal{R}\right) / I_{\mathcal{P}_{n}}$, as usual, we let $\leq$ be inherited by the ordering $\leq$ on $\mathcal{L}\left(\mathcal{X}^{n} \times \mathcal{R}\right)$ :

$$
\tilde{f} \leq \tilde{g} \Leftrightarrow(\exists f \in \tilde{f}, \exists g \in \tilde{g}) f \leq g
$$

for all $\tilde{f}$ and $\tilde{g}$ in $\mathcal{L}\left(\mathcal{X}^{n} \times \mathcal{R}\right) / I_{\mathcal{P}_{n}}$, turning $\mathcal{L}\left(\mathcal{X}^{n} \times \mathcal{R}\right) / I_{\mathcal{P}_{n}}$ into an ordered linear space. It turns out that this vector ordering on $\mathcal{L}\left(\mathcal{X}^{n} \times \mathcal{R}\right) / I_{\mathcal{P}_{n}}$ also can be represented elegantly using the map $\tilde{\mathrm{H}}_{n}$ :

Proposition 6. Consider any $\tilde{f}$ and $\tilde{g}$ in $\mathcal{L}\left(\mathcal{X}^{n} \times \mathcal{R}\right) / I_{\mathcal{P}_{n}}$, then $\tilde{f} \leq \tilde{g}$ if and only if $\tilde{\mathrm{H}}_{n}(\tilde{f}) \leq$ $\tilde{\mathrm{H}}_{n}(\tilde{g})$.

Proof. For necessity, assume that $\tilde{f} \leq \tilde{g}$. Then, by Equation $[10, f \leq g$ for some $f$ in $\tilde{f}$ and $g$ in $\tilde{g}$. Consider any $m$ in $\mathcal{N}^{n}$, and infer that $\mathrm{H}_{n}(f)(m, \bullet)=\frac{1}{\left(\begin{array}{c}n \\ m\end{array}\right)} \sum_{y \in[m]} f(y, \bullet) \leq$ $\frac{1}{\left(\begin{array}{c}n \\ m\end{array}\right)} \sum_{y \in[m]} g(y, \bullet)=\mathrm{H}_{n}(g)(m, \bullet)$. Then $\mathrm{H}_{n}(f) \leq \mathrm{H}_{n}(g)$, and therefore, by Equation (7), indeed $\tilde{\mathrm{H}}_{n}(\tilde{f}) \leq \tilde{\mathrm{H}}_{n}(\tilde{g})$.

For sufficiency, assume that $\tilde{\mathrm{H}}_{n}(\tilde{f}) \leq \tilde{\mathrm{H}}_{n}(\tilde{g})$. Then, by Equation (7) and Proposition 4 $\mathrm{H}_{n}(f) \leq \mathrm{H}_{n}(g)$ for all $f$ in $\tilde{f}$ and $g$ in $\tilde{g}$. Consider any $f$ in $\tilde{f}$ and $g$ in $\tilde{g}$ and let $f^{\prime}:=$ $\operatorname{inv}_{\mathcal{P}_{n}}(f)$ and $g^{\prime}:=\operatorname{inv}_{\mathcal{P}_{n}}(g)$. Then $\operatorname{inv}_{\mathcal{P}_{n}}\left(f^{\prime}\right)=\operatorname{inv}_{\mathcal{P}_{n}}(f)$ and $\operatorname{inv}_{\mathcal{P}_{n}}\left(g^{\prime}\right)=\operatorname{inv}_{\mathcal{P}_{n}}(g)$ by Proposition 2(ii), so Proposition 4 implies that $f^{\prime} \in \tilde{f}$ and $g^{\prime} \in \tilde{f}$, and therefore $\mathrm{H}_{n}\left(f^{\prime}\right) \leq$ 
$\mathrm{H}_{n}\left(g^{\prime}\right)$. Then, by Equations [6] and [5], $\frac{1}{\left(\begin{array}{c}n \\ m\end{array}\right)} \sum_{y \in[m]} f^{\prime}(y, \bullet) \leq \frac{1}{\left(\begin{array}{c}n \\ m\end{array}\right)} \sum_{y \in[m]} g^{\prime}(y, \bullet)$ for every $m$ in $\mathcal{N}^{n}$. But $f^{\prime}$ and $g^{\prime}$ are constant on every $[m]$, so $f^{\prime}(y, \bullet) \leq g^{\prime}(y, \bullet)$ for every $y$ in $[m]$ and every $m$ in $\mathcal{N}^{n}$. Then $f^{\prime} \leq g^{\prime}$, and therefore indeed $\tilde{f} \leq \tilde{g}$.

Propositions 5 and 6 imply that $\tilde{\mathrm{H}}_{n}$ is a linear order isomorphism between $\mathcal{L}\left(\mathcal{X}^{n} \times \mathcal{R}\right) / I_{\mathcal{P}_{n}}$ and $\mathcal{L}\left(\mathcal{N}^{n} \times \mathcal{R}\right)$, and therefore, every coherent choice function on $\mathcal{L}\left(\mathcal{X}^{n} \times \mathcal{R}\right) / I_{\mathcal{P}_{n}}$ can be identified with a coherent choice function on $\mathcal{L}\left(\mathcal{N}^{n} \times \mathcal{R}\right)$. We will use this interesting conclusion in the next section.

4.3. A representation theorem. Now that the preparatory work is done, we are ready to establish our representation for coherent and exchangeable choice functions.

Theorem 7 (Finite representation). Consider any choice function $C_{n}$ on $\mathcal{L}\left(\mathcal{X}^{n} \times \mathcal{R}\right)$. Then $C_{n}$ is exchangeable if and only if there is a unique representing choice function $\tilde{C}$ on $\mathcal{L}\left(\mathcal{N}^{n} \times \mathcal{R}\right)$ such that

$$
C_{n}(A)=\left\{f \in A: \mathrm{H}_{n}(f) \in \tilde{C}\left(\mathrm{H}_{n}(A)\right)\right\} \text { for all } A \text { in } \mathcal{Q}\left(\mathcal{L}\left(\mathcal{X}^{n} \times \mathcal{R}\right)\right) \text {. }
$$

Furthermore, in that case, $\tilde{C}$ is given by $\tilde{C}\left(\mathrm{H}_{n}(A)\right)=\mathrm{H}_{n}\left(C_{n}(A)\right)$ for all $A$ in $\mathcal{Q}\left(\mathcal{L}\left(\mathcal{X}^{n} \times \mathcal{R}\right)\right)$. Finally, $C_{n}$ is coherent if and only if $\tilde{C}$ is. We call $\tilde{C}$ the count representation of $C_{n}$.

Similarly, consider any set of desirable gambles $D_{n} \subseteq \mathcal{L}\left(\mathcal{X}^{n} \times \mathcal{R}\right)$. Then $D_{n}$ is exchangeable if and only if there is a unique representing set of desirable gambles $\tilde{D} \subseteq \mathcal{L}\left(\mathcal{N}^{n} \times \mathcal{R}\right)$ such that $D_{n}=\bigcup \tilde{\mathrm{H}}_{n}^{-1}(\tilde{D})$. Furthermore, in that case, $\tilde{D}$ is given by $\tilde{D}=\mathrm{H}_{n}\left(D_{n}\right)$. Finally, $D_{n}$ is coherent if and only if $\tilde{D}$ is. We call $\tilde{D}$ the count representation of $D_{n}$.

Proof. We begin with the representation of choice functions. For the first statement, note that $C_{n}$ is exchangeable is equivalent to $C_{n}$ is compatible with $I_{\mathcal{P}_{n}}$, by Definition 6. Equivalently, by Definition 5, there is some representing choice function $C^{\prime}$ on $\mathcal{L}\left(\mathcal{X}^{n} \times \mathcal{R}\right) / I_{\mathcal{P}_{n}}$ such that $C_{n}(A)=\left\{f \in A:[f] \in C^{\prime}\left(A / I_{\mathcal{P}_{n}}\right)\right\}$ for all $A$ in $\mathcal{Q}\left(\mathcal{L}\left(\mathcal{X}^{n} \times \mathcal{R}\right)\right)$. We use the linear order isomorphism $\tilde{\mathrm{H}}_{n}$ to define a choice function $\tilde{C}$ on $\mathcal{L}\left(\mathcal{N}^{n} \times \mathcal{R}\right)$ : we let $[f] \in C^{\prime}\left(A / I_{\mathcal{P}_{n}}\right) \Leftrightarrow$ $\tilde{\mathrm{H}}_{n}([f]) \in \tilde{C}\left(\tilde{\mathrm{H}}_{n}\left(A / I_{\mathcal{P}_{n}}\right)\right)$ for all $f$ in $\mathcal{L}\left(\mathcal{X}^{n} \times \mathcal{R}\right)$ and $A$ in $\mathcal{Q}\left(\mathcal{L}\left(\mathcal{X}^{n} \times \mathcal{R}\right)\right)$. Since $f \in[f]$, use Proposition 4 and Equation (7) to infer that $\tilde{\mathrm{H}}_{n}([f])=\mathrm{H}_{n}(f)$. Similarly, infer that $\tilde{\mathrm{H}}_{n}\left(A / I_{\mathcal{P}_{n}}\right)=\left\{\tilde{\mathrm{H}}_{n}([g]): g \in A\right\}=\left\{\mathrm{H}_{n}(g): g \in A\right\}=\mathrm{H}_{n}(A)$, so $[f] \in C^{\prime}\left(A / I_{\mathcal{P}_{n}}\right) \Leftrightarrow \mathrm{H}_{n}(f) \epsilon$ $\tilde{C}\left(\mathrm{H}_{n}(A)\right)$. Then indeed

$$
C_{n}(A)=\left\{f \in A: \mathrm{H}_{n}(f) \in \tilde{C}\left(\mathrm{H}_{n}(A)\right)\right\} \text { for all } A \text { in } \mathcal{Q}\left(\mathcal{L}\left(\mathcal{X}^{n} \times \mathcal{R}\right)\right) .
$$

To show that $\tilde{C}$ is unique, use that $C^{\prime}$ is unique and $\tilde{\mathrm{H}}_{n}$ is a bijection.

For the second statement, consider any $A$ in $\mathcal{Q}\left(\mathcal{L}\left(\mathcal{X}^{n} \times \mathcal{R}\right)\right)$ and infer, using the definition of $\tilde{C}$, that $\tilde{C}\left(\tilde{\mathrm{H}}_{n}\left(A / I_{\mathcal{P}_{n}}\right)\right)=\tilde{\mathrm{H}}_{n}\left(C^{\prime}\left(A / I_{\mathcal{P}_{n}}\right)\right)$, and therefore $\tilde{C}\left(\mathrm{H}_{n}(A)\right)=\tilde{\mathrm{H}}_{n}\left(C^{\prime}\left(A / I_{\mathcal{P}_{n}}\right)\right)$ by Equation (7). By compatibility, we have that $C^{\prime}\left(A / I_{\mathcal{P}_{n}}\right)=C_{n}(A) / I_{\mathcal{P}_{n}}$, so we find that $\tilde{\mathrm{H}}_{n}\left(C^{\prime}\left(A / I_{\mathcal{P}_{n}}\right)\right)=\tilde{\mathrm{H}}_{n}\left(C_{n}(A) / I_{\mathcal{P}_{n}}\right)=\mathrm{H}_{n}\left(C_{n}(A)\right)$, by Equation (7), and therefore indeed $\tilde{C}\left(\mathrm{H}_{n}(A)\right)=\mathrm{H}_{n}\left(C_{n}(A)\right)$.

For the third statement, the compatibility with $I_{\mathcal{P}_{n}}$ guarantees that $C_{n}$ is coherent if and only if its representing choice function $C^{\prime}$ on $\mathcal{L}\left(\mathcal{X}^{n} \times \mathcal{R}\right) / I_{\mathcal{P}_{n}}$ is coherent. But since $\tilde{C}$ is defined from $C^{\prime}$ using the linear order isomorphism $\mathrm{H}_{n}$, we have immediately that $\tilde{C}$ is coherent if and only if $C^{\prime}$ is coherent.

The representation of sets of desirable gambles is a trivial extension to vector-valued gambles of the proof given in Reference [6, Theorem 17].

The number of occurrences of any outcome in a sequence $\left(x_{1}, \ldots, x_{n}\right)$ is fixed by its count vector $m$ in $\mathcal{N}^{n}$. If we impose an exchangeability assessment on it, then we see, using Theorem 7, that the joint model on $\mathcal{X}^{n}$ is characterised by a model on $\mathcal{L}\left(\mathcal{N}^{n} \times \mathcal{R}\right)$. So an 
exchangeable choice function $C_{n}$ essentially represents preferences between gambles on the unknown composition $m$ of an urn with $n$ balls of types $\mathcal{X}$ : the choice $C_{n}(A)$ between the gambles in $A$ is based on $m$.

This representation result immediately translates to rejection functions. A rejection function $R$ on $\mathcal{L}\left(\mathcal{X}^{n} \times \mathcal{R}\right)$ is exchangeable if and only if there is a unique representing rejection function $\tilde{R}$ on $\mathcal{L}\left(\mathcal{N}^{n} \times \mathcal{R}\right)$, given by $\tilde{R}\left(\mathrm{H}_{n}(A)\right)=\mathrm{H}_{n}(R(A))$ for all $A$ in $\mathcal{Q}\left(\mathcal{L}\left(\mathcal{X}^{n} \times\right.\right.$ $\mathcal{R})$ ), that represents $R$, and that is coherent if and only if $R$ is.

4.4. Conditioning exchangeable models. We use the same notation and ideas as in Reference [6, Section 4.4] for desirability, and generalise it to choice models.

Consider an exchangeable and coherent choice function $C$ on $\mathcal{L}\left(\mathcal{X}^{n} \times \mathcal{R}\right)$, and assume that we have observed the values $\check{x}:=\left(\check{x}_{1}, \ldots, \check{x}_{\breve{n}}\right)$ of the first $\check{n}$ variables $X_{1}, \ldots, X_{\breve{n}}$, and that we want to make inferences about the remaining $\hat{n}:=n-\check{n}$ variables. To do this, we simply condition the choice function $C$ on the singleton $\{\check{x}\}:^{8}$

$$
f \in C \mid \check{x}(A) \Leftrightarrow \mathbb{I}_{\{\check{x}\}} f \in C\left(\mathbb{I}_{\{\check{x}\}} A\right) \text { for all } A \text { in } \mathcal{Q}\left(\mathcal{L}\left(\mathcal{X}^{\hat{n}} \times \mathcal{R}\right)\right) \text { and } f \text { in } A,
$$

following the general discussion in Section 3

Proposition 8. Consider any $\check{x}$ in $\mathcal{X}^{\check{n}}$ and any coherent and exchangeable choice function $C$ on $\mathcal{L}\left(\mathcal{X}^{n} \times \mathcal{R}\right)$. Then $C \mid \breve{x}$ is a coherent and exchangeable choice function on $\mathcal{L}\left(\mathcal{X}^{\hat{n}} \times \mathcal{R}\right)$.

Proof. That $C] \check{x}$ is coherent, follows from Proposition 1 . It therefore suffices to show that $C\rfloor \check{x}$ is exchangeable. By Definition 6, we need to show that $C\rfloor \breve{x}$ is compatible with $I_{\mathcal{P}_{\hat{n}}}$. By Equation (1), this is equivalent to

$$
\left.\left.\left(\forall g \in I_{\mathcal{P}_{\hat{n}}}\right)\left(\forall A \in \mathcal{Q}\left(\mathcal{L}\left(\mathcal{X}^{\hat{n}} \times \mathcal{R}\right)\right)\right)(\{0, g\} \subseteq A \Rightarrow(0 \in C] \breve{x}(A) \Leftrightarrow g \in C] \breve{x}(A)\right)\right) .
$$

So consider any $g$ in $I_{\mathcal{P}_{\hat{n}}}$ and any $A$ in $\mathcal{Q}\left(\mathcal{L}\left(\mathcal{X}^{\hat{n}} \times \mathcal{R}\right)\right)$ such that $\{0, g\} \subseteq A$. It suffices to show that then $0 \in C\left(\mathbb{I}_{\{\check{x}\}} A\right) \Leftrightarrow \mathbb{I}_{\{\check{x}\}} g \in C\left(\mathbb{I}_{\{\check{x}\}} A\right)$. Since $C$ is exchangeable-compatible with $I_{\mathcal{P}_{n}}$ - and again using Equation (1), this will be the case if we can prove that $\mathbb{I}_{\{\breve{x}\}} g$ belongs to $I_{\mathcal{P}_{n}}$. To show this, since $g \in I_{\mathcal{P}_{\hat{n}}}$, observe by Equation (3) that $g=\sum_{i=1}^{m} \lambda_{i}\left(f_{i}-\hat{\pi}_{i}^{t} f_{i}\right)$ for some $m$ in $\mathbb{N}, \lambda_{1}, \ldots, \lambda_{m}$ in $\mathbb{R}, f_{1}, \ldots, f_{m}$ in $\mathcal{L}\left(\mathcal{X}^{\hat{n}} \times \mathcal{R}\right)$, and $\hat{\pi}_{1}, \ldots, \hat{\pi}_{m}$ in $\mathcal{P}_{\hat{n}}$. For every $i$ in $\{1, \ldots, m\}$, let $\pi_{i} \in \mathcal{P}_{n}$ be defined as

$$
\pi_{i}(k):=\left\{\begin{array}{ll}
k & \text { if } k \in\{1, \ldots, \breve{n}\} \\
\check{n}+\hat{\pi}_{i}(k-\check{n}) & \text { if } k \in\{\check{n}+1, \ldots, n\}
\end{array} \text { for all } k \text { in }\{1, \ldots, n\},\right.
$$

being a permutation that is constant on the first $\check{n}$ components. Then $\mathbb{I}_{\{\check{x}\}} \hat{\pi}_{i}^{t} f_{i}=\pi_{i}^{t}\left(\mathbb{I}_{\{\check{x}\}} f_{i}\right)$ for every $i$ in $\{1, \ldots, m\}$, whence

$$
\mathbb{I}_{\{\check{x}\}} g=\sum_{i=1}^{m} \lambda_{i}\left(\mathbb{I}_{\{\check{x}\}} f_{i}-\mathbb{I}_{\{\check{x}\}} \hat{\pi}_{i}^{t} f_{i}\right)=\sum_{i=1}^{m} \lambda_{i}\left(\mathbb{I}_{\{\check{x}\}} f_{i}-\pi_{i}^{t}\left(\mathbb{I}_{\{\check{x}\}} f_{i}\right)\right),
$$

so $\mathbb{I}_{\{\check{x}\}} g$ indeed belongs to $I_{\mathcal{P}_{n}}$.

\footnotetext{
${ }^{8}$ Actually, the conditioning event is $\{\check{x}\} \times \mathcal{X}^{\hat{n}}$. In order to streamline the argument and the notation, we will identify $\{\check{x}\}$ with $\{\check{x}\} \times \mathcal{X}^{\hat{n}}$, which comes down to identifying gambles (and in particular indicators) with their cylindrical extensions, as is commonly done in the literature [4] 5]. See Section 5 for more information about the cylindrical extension. Formally, the conditional choice function $C] \breve{x}$ is defined on $\mathcal{Q}\left(\mathcal{L}\left(\{\breve{x}\} \times \mathcal{X}^{\hat{n}} \times \mathcal{R}\right)\right)$, but since any gamble $f$ in $\mathcal{L}\left(\{\check{x}\} \times \mathcal{X}^{\hat{n}} \times \mathcal{R}\right)$ can be uniquely identified with the gamble $f(\check{x}, \bullet)$ in $\mathcal{L}\left(\mathcal{X}^{\hat{n}} \times \mathcal{R}\right)$, we can equivalently define $C\rfloor \breve{x}$ on $\mathcal{Q}\left(\mathcal{L}\left(\mathcal{X}^{\hat{n}} \times \mathcal{R}\right)\right)$.
} 
We also introduce another type of conditioning, where we observe a count vector $\check{m}$ in $\mathcal{N}^{\check{n}}$, and we condition the choice function $C$ on all the possible sequences $[\check{m}]$ with these counts, to obtain $C][\check{m}]$. The domain of this conditional choice function is $\mathcal{Q}\left(\mathcal{L}\left([\check{m}] \times \mathcal{X}^{\hat{n}} \times \mathcal{R}\right)\right)$, and we are interested in its marginal choice function $\left.\operatorname{marg}_{\hat{n}}(C][\check{m}]\right)$ about the $\hat{n}$ remaining variables $X_{\check{n}+1}, \ldots, X_{n}$, which is defined as

$$
\left.\left.\operatorname{marg}_{\hat{n}}(C][\check{m}]\right)(A):=C\right][\check{m}](A) \text { for all } A \text { in } \mathcal{Q}\left(\mathcal{L}\left(\mathcal{X}^{\hat{n}} \times \mathcal{R}\right)\right) .
$$

Therefore

$$
\left.f \in\left(\operatorname{marg}_{\hat{n}}(C][\check{m}]\right)\right)(A) \Leftrightarrow \mathbb{I}_{[\check{m}]} f \in C\left(\mathbb{I}_{[\check{m}]} A\right) \text {, for all } A \text { in } \mathcal{Q}\left(\mathcal{L}\left(\mathcal{X}^{\hat{n}} \times \mathcal{R}\right)\right) \text { and } f \text { in } A \text {. }
$$

To simplify the notation, let $\left.C\rfloor \breve{m}:=\operatorname{marg}_{\hat{n}}(C][\check{m}]\right)$. Interestingly, the count vector $\check{m}$ for an observed sample $\check{x}$ is a sufficient statistic in the sense that it extracts from $\check{x}$ all the information that is needed to characterise the conditional model:

Proposition 9 (Sufficiency of the observed count vector). Consider any coherent and exchangeable choice function $C$ on $\mathcal{L}\left(\mathcal{X}^{n} \times \mathcal{R}\right)$, and any $\check{x}$ and $\check{y}$ in $\mathcal{X}^{\check{n}}$. If $[\check{x}]=[\check{y}]$, or in other words, if $T(\check{x})=T(\check{y})=: \check{m}$, then $C \mid \check{x}=C] \breve{y}=C \mid \check{m}$.

Proof. From $[\check{x}]=[\check{y}]$, infer that $\check{y}=\check{\pi} \check{x}$ for some $\check{\pi}$ in $\mathcal{P}_{\check{n}}$, and from $T(\check{x})=T(\check{y})=\check{m}$, infer that $\check{x} \in[\check{m}]$ and $\check{y} \in[\check{m}]$. We will first show the intermediate result that then

$$
\mathrm{H}_{n}\left(\mathbb{I}_{\{\check{x}\}} A\right)=\mathrm{H}_{n}\left(\mathbb{I}_{\{\check{y}\}} A\right)=\frac{1}{|[\check{m}]|} \mathrm{H}_{n}\left(\mathbb{I}_{[\check{m}]} A\right) \text { for all } A \text { in } \mathcal{Q}\left(\mathcal{L}\left(\mathcal{X}^{\hat{n}} \times \mathcal{R}\right)\right) .
$$

Consider any $A$ in $\mathcal{Q}\left(\mathcal{L}\left(\mathcal{X}^{\hat{n}} \times \mathcal{R}\right)\right)$. Define the permutation $\pi$ in $\mathcal{P}_{n}$ as

$$
\pi(k):=\left\{\begin{array}{ll}
\check{\pi}^{-1}(k) & \text { if } k \in\{1, \ldots, \breve{n}\} \\
k & \text { if } k \in\{\check{n}+1, \ldots, n\}
\end{array} \text { for all } k \text { in }\{1, \ldots, n\} .\right.
$$

We claim that then $\pi^{t}\left(\mathbb{I}_{\{\check{x}\}} f\right)=\mathbb{I}_{\{\check{y}\}} f$ for all $f$ in $A$. To establish this, consider any $\check{z}=$ $\left(\check{z}_{1}, \ldots, \check{z}_{n}\right)$ in $\mathcal{X}^{n}$ and any $r$ in $\mathcal{R}$, and observe that indeed

$$
\begin{aligned}
\left(\pi^{t}\left(\mathbb{I}_{\{\check{x}\}} f\right)\right)(\check{z}, r)=\mathbb{I}_{\{\check{x}\}} f(\pi(\check{z}), r) & = \begin{cases}f\left(\pi\left(\check{z}_{\check{n}+1}, \ldots, \check{z}_{n}\right), r\right) & \text { if } \pi\left(\check{z}_{1}, \ldots, \check{z}_{\check{n}}\right)=\check{x} \\
0 & \text { if } \pi\left(\check{z}_{1}, \ldots, \check{z}_{\check{n}}\right) \neq \check{x}\end{cases} \\
& = \begin{cases}f\left(\check{z}_{\check{n}+1}, \ldots, \check{z}_{n}, r\right) & \text { if }\left(\check{z}_{1}, \ldots, \check{z}_{\check{n}}\right)=\check{\pi} \check{x} \\
0 & \text { if }\left(\check{z}_{1}, \ldots, \check{z}_{\check{n}}\right)=\check{\pi} \check{x}\end{cases} \\
& =\mathbb{I}_{\{\check{\pi} \check{x}\}} f(\check{z}, r)=\mathbb{I}_{\{\breve{y}\}} f(\check{z}, r) .
\end{aligned}
$$

So we see that $\pi^{t}\left(\mathbb{I}_{\{\check{x}\}} f\right)=\mathbb{I}_{\{\check{y}\}} f$ and therefore $\mathbb{I}_{\{\check{x}\}} f-\mathbb{I}_{\{\check{y}\}} f \in I_{\mathcal{P}_{n}}$, whence $\left[\mathbb{I}_{\{\check{x}\}} f\right]=$ $\left[\mathbb{I}_{\{\check{y}\}} f\right]$. By Proposition 4 , observe that then $\mathrm{H}_{n}\left(\mathbb{I}_{\{\check{x}\}} f\right)=\mathrm{H}_{n}\left(\mathbb{I}_{\{\check{y}\}} f\right)$ for all $f$ in $A$, whence $\mathrm{H}_{n}\left(\mathbb{I}_{\{\check{x}\}} A\right)=\mathrm{H}_{n}\left(\mathbb{I}_{\{\check{y}\}} A\right)$. To show that this is also equal to $\frac{1}{[\check{m}] \mid} \mathrm{H}_{n}\left(\mathbb{I}_{[\check{m}]} A\right)$, observe that $[\check{m}]=\left\{\check{\varpi} \check{x}: \check{\varpi} \in \mathcal{P}_{\check{n}}\right\}$, and therefore for any $\check{z}$ in $[\check{m}]$, we can select a $\check{\pi}_{\check{z}}$ in $\mathcal{P}_{\check{n}}$ such that $\check{\pi}_{\check{z}} \check{x}=\check{z}$. With this $\check{\pi}_{\check{z}}$ we construct a permutation $\pi_{\check{z}}$ in the manner described above, which satisfies $\pi_{\check{z}}^{t}\left(\mathbb{I}_{\{\check{x}\}} f\right)=\mathbb{I}_{\{\check{z}\}} f$ for every $f$ in $A$. Use $\mathbb{I}_{[\check{m}]} f=\sum_{\check{z} \in[\check{m}]} \mathbb{I}_{\{\check{z}\}} f$ for every $f$ in $A$ to infer that indeed $\mathrm{H}_{n}\left(\mathbb{I}_{[\check{m}]} A\right)=\mathrm{H}_{n}\left(\sum_{\check{z} \in[\check{m}]} \mathbb{I}_{\{\check{z}\}} A\right)=\sum_{\check{z} \in[\check{m}]} \mathrm{H}_{n}\left(\mathbb{I}_{\{\check{z}\}} A\right)=|[\check{m}]| \mathrm{H}_{n}\left(\mathbb{I}_{\{\check{x}\}} A\right)$, where the last equation holds because we have already shown that $\mathrm{H}_{n}\left(\mathbb{I}_{\{\check{z}\}} A\right)=\mathrm{H}_{n}\left(\mathbb{I}_{\{\check{x}\}} A\right)$ for every $\check{z}$ in $[\check{x}]=[\check{m}]$.

Now we are ready to show that $C] \breve{x}=C] \breve{y}=C\rfloor \breve{m}$. Since they are coherent, it suffices to show that

$$
0 \in C\rfloor \breve{x}(A) \Leftrightarrow 0 \in C\rfloor \breve{y}(A) \Leftrightarrow 0 \in C\rfloor \breve{m}(A), \text { for all } A \text { in } \mathcal{Q}\left(\mathcal{L}\left(\mathcal{X}^{\hat{n}} \times \mathcal{R}\right)\right),
$$


and therefore, taking into account that $\left.C] \check{m}=\operatorname{marg}_{\hat{n}}(C][\check{m}]\right)$, that

$$
0 \in C\left(\mathbb{I}_{\{\check{x}\}} A\right) \Leftrightarrow 0 \in C\left(\mathbb{I}_{\{\check{y}\}} A\right) \Leftrightarrow 0 \in C\left(\mathbb{I}_{[\check{m}]} A\right) \text {, for all } A \text { in } \mathcal{Q}\left(\mathcal{L}\left(\mathcal{X}^{\hat{n}} \times \mathcal{R}\right)\right) \text {. }
$$

Because $C$ is exchangeable, by Theorem 7 it suffices to show that

$$
0 \in C\left(\mathrm{H}_{n}\left(\mathbb{I}_{\{\check{x}\}} A\right)\right) \Leftrightarrow 0 \in C\left(\mathrm{H}_{n}\left(\mathbb{I}_{\{\check{y}\}} A\right)\right) \Leftrightarrow 0 \in C\left(\mathrm{H}_{n}\left(\mathbb{I}_{[\check{m}]} A\right)\right),
$$

for all $A$ in $\mathcal{Q}\left(\mathcal{L}\left(\mathcal{X}^{\hat{n}} \times \mathcal{R}\right)\right)$. But we have shown above that $\mathrm{H}_{n}\left(\mathbb{I}_{\{\check{x}\}} A\right)=\mathrm{H}_{n}\left(\mathbb{I}_{\{\check{y}\}} A\right)=$ $\frac{1}{[[\check{m}] \mid} \mathrm{H}_{n}\left(\mathbb{I}_{[\check{m}]} A\right)$, so taking coherence [and more specifically, Axiom $\mathrm{C} 4 \mathrm{a}$ into account, this is indeed the case.

4.5. Finite representation in terms of polynomials. In Section 5 , we will prove a similar representation theorem for infinite sequences. Since it no longer makes sense to count in such sequences, we first need to find a equivalent representation theorem in terms of something that does not depend on counts. More specifically, we need, for every $n$ in $\mathbb{N}$ another order-isomorphic linear space to $\mathcal{L}\left(\mathcal{X}^{n} \times \mathcal{R}\right) / I_{\mathcal{P}_{n}}$ that allows for embedding: the linear space for any $n_{1} \leq n_{2}$ (both in $\mathbb{N}$ ) must be a subspace of the one for $n_{2}$.

All the relevant maps in this section have been introduced by De Cooman et al. [6, 7]. We use their ideas and work with polynomials on the $\mathcal{X}$-simplex

$$
\Sigma_{\mathcal{X}}:=\left\{\theta \in \mathbb{R}^{\mathcal{X}}: \theta \geq 0, \sum_{x \in \mathcal{X}} \theta_{x}=1\right\} .
$$

We consider the special subset $\mathcal{V}\left(\Sigma_{\mathcal{X}} \times \mathcal{R}\right)$ of $\mathcal{L}\left(\Sigma_{\mathcal{X}} \times \mathcal{R}\right): \mathcal{V}\left(\Sigma_{\mathcal{X}} \times \mathcal{R}\right)$ are the polynomial vector-valued gambles on $\Sigma_{\mathcal{X}} \times \mathcal{R}$, which are those gambles $h$ such that for every $r$ in $\mathcal{R}$, $h(\bullet, r)$ is the restriction to $\Sigma_{\mathcal{X}}$ of a multivariate polynomial $p_{r}$ on $\mathbb{R}^{\mathcal{X}}$, in the sense that $h(\theta, r)=p_{r}(\theta)$ for all $\theta$ in $\Sigma_{\mathcal{X}}$. We call $p_{r}$ then a representation of $h(\bullet, r)$. It will be useful to introduce a notation for polynomial vector-valued gambles with fixed degree $n$ in $\mathbb{N}$ : $\mathcal{V}^{n}\left(\Sigma_{\mathcal{X}} \times \mathcal{R}\right)$ is the collection of all polynomial vector-valued gambles $h$ such that for every $r$ in $\mathcal{R}, h(\bullet, r)$ has at least one representation whose degree is not higher than $n$. As shown in References [4, 6], both $\mathcal{V}\left(\Sigma_{\mathcal{X}} \times \mathcal{R}\right)$ and $\mathcal{V}^{n}\left(\Sigma_{\mathcal{X}} \times \mathcal{R}\right)$ are linear subspaces of $\mathcal{L}\left(\Sigma_{\mathcal{X}} \times \mathcal{R}\right)$, and, as we wanted, for $n_{1} \leq n_{2}, \mathcal{V}^{n_{1}}\left(\Sigma_{\mathcal{X}} \times \mathcal{R}\right)$ is a subspace of $\mathcal{V}^{n_{2}}\left(\Sigma_{\mathcal{X}} \times \mathcal{R}\right)$.

Special polynomial gambles are the Bernstein gambles:

Definition 7 (Bernstein gambles). Consider any $n$ in $\mathbb{N}$ and any $m$ in $\mathcal{N}^{n}$. Define the Bernstein basis polynomial $B_{m}$ on $\mathbb{R}^{\mathcal{X}}$ as $B_{m}(\theta):=\left(\begin{array}{c}n \\ m\end{array}\right) \prod_{x \in \mathcal{X}} \theta_{x}^{m_{x}}$ for all $\theta$ in $\mathbb{R}^{\mathcal{X}}$. The restriction of $B_{m}$ to $\Sigma_{\mathcal{X}}$ is called a Bernstein gamble, which we also denote as $B_{m}$.

As mentioned by De Cooman and Quaeghebeur [6] and proved explicitly by De Bock et al. [4], the set of all Bernstein gambles constitutes a basis for the linear space $\mathcal{V}^{n}\left(\Sigma_{\mathcal{X}}\right)$ :

Proposition 10 ([6, Appendix B], [4, Proposition 14]). Consider any $n$ in $\mathbb{N}$. The set of Bernstein gambles $\left\{B_{m}: m \in \mathcal{N}^{n}\right\}$ constitutes a basis for the linear space $\mathcal{V}^{n}\left(\Sigma_{\mathcal{X}}\right)$. Therefore, each element $p$ of $\mathcal{V}^{n}\left(\Sigma_{\mathcal{X}} \times \mathcal{R}\right)$ can be uniquely written as $p(\theta, r)=\sum_{m \in \mathcal{N}^{n}} \alpha(m, r) B_{m}(\theta)$ for every $\theta$ in $\Sigma_{\mathcal{X}}$ and $r$ in $\mathcal{R}$.

As we have seen, to preserve coherence between two ordered linear spaces, we need a linear order isomorphism. So we wonder whether there is one between $\mathcal{L}\left(\mathcal{X}^{n} \times \mathcal{R}\right) / I_{\mathcal{P}_{n}}$ and $\mathcal{V}^{n}\left(\Sigma_{\mathcal{X}} \times \mathcal{R}\right)$. In Section 4.2 we have seen that there is one between $\mathcal{L}\left(\mathcal{X}^{n} \times \mathcal{R}\right) / I_{\mathcal{P}_{n}}$ and $\mathcal{L}\left(\mathcal{N}^{n} \times \mathcal{R}\right)$, namely $\tilde{\mathrm{H}}_{n}$. Therefore, it suffices to find one between $\mathcal{L}\left(\mathcal{N}^{n} \times \mathcal{R}\right)$ and $\mathcal{V}^{n}\left(\Sigma_{\mathcal{X}} \times \mathcal{R}\right)$. Consider the map

$$
\operatorname{CoM}_{n}: \mathcal{L}\left(\mathcal{N}^{n} \times \mathcal{R}\right) \rightarrow \mathcal{V}^{n}\left(\Sigma_{\mathcal{X}} \times \mathcal{R}\right): h \mapsto \sum_{m \in \mathcal{N}^{n}} h(m, \bullet) B_{m}
$$


Before we can establish that $\mathrm{CoM}_{n}$ is a linear order isomorphism, we need to provide the linear space $\mathcal{V}^{n}\left(\Sigma_{\mathcal{X}} \times \mathcal{R}\right)$ with an order $\leq_{B}^{n}$. To this end, we use the proper cone $\{0\} \cup$ $\operatorname{posi}\left(\left\{B_{m}: m \in \mathcal{N}^{n}\right\}\right)$ :

$$
h_{1} \leq_{B}^{n} h_{2} \Leftrightarrow(\forall r \in \mathcal{R}) h_{2}(\bullet, r)-h_{1}(\cdot, r) \in\{0\} \cup \operatorname{posi}\left(\left\{B_{m}: m \in \mathcal{N}^{n}\right\}\right),
$$

for all $h_{1}$ and $h_{2}$ in $\mathcal{V}^{n}\left(\Sigma_{\mathcal{X}} \times \mathcal{R}\right)$.

The following proposition is essentially due to De Cooman and Quaeghebeur [6]: it suffices to apply their result point-wise, for every $r$ in $\mathcal{R}$.

Proposition 11 ([6, Section 4.9], [4, Section 4.5]). Consider any $n$ in $\mathbb{N}$. Then the map $\mathrm{CoM}_{n}$ is a linear order isomorphism between the ordered linear spaces $\mathcal{L}\left(\mathcal{N}^{n} \times \mathcal{R}\right)$ and $\mathcal{V}^{n}\left(\Sigma_{\mathcal{X}} \times \mathcal{R}\right)$.

The linear order isomorphism $\mathrm{CoM}_{n}$ helps us to define a linear order isomorphism between the linear spaces $\mathcal{L}\left(\mathcal{X}^{n} \times \mathcal{R}\right)$ and $\mathcal{V}^{n}\left(\Sigma_{\mathcal{X}} \times \mathcal{R}\right)$, a final tool needed for a representation theorem in terms of polynomial gambles. Indeed, consider the map $\mathrm{M}_{n}:=\mathrm{CoM}_{n} \circ \mathrm{H}_{n}$ :

$$
\mathbf{M}_{n}: \mathcal{L}\left(\mathcal{X}^{n} \times \mathcal{R}\right) \rightarrow \mathcal{V}^{n}\left(\Sigma_{\mathcal{X}} \times \mathcal{R}\right): f \mapsto \mathrm{M}_{n}(f)
$$

where $\mathbf{M}_{n}(f)(\theta, r):=\mathbf{M}_{n}(f(\bullet, r) \mid \theta)$ for all $\theta$ in $\Sigma_{\mathcal{X}}$ and $r$ in $\mathcal{R} . \mathbf{M}_{n}(\bullet \mid \theta)$ is the linear expectation operator associated with the multinomial distribution whose parameters are $n$ and $\theta$, and is for every $g$ in $\mathcal{L}\left(\mathcal{X}^{n}\right)$ given by $\mathbf{M}_{n}(g \mid \theta):=\sum_{m \in \mathcal{N}^{n}} \sum_{y \in[m]} g(y) \prod_{x \in \mathcal{X}} \theta_{x}^{m_{x}}$. We introduce its version

$$
\tilde{\mathrm{M}}_{n}:=\mathrm{CoM}_{n} \circ \tilde{\mathrm{H}}_{n},
$$

mapping $\mathcal{L}\left(\mathcal{X}^{n} \times \mathcal{R}\right) / I_{\mathcal{P}_{n}}$ to $\mathcal{V}^{n}\left(\Sigma_{\mathcal{X}} \times \mathcal{R}\right)$. $\tilde{\mathrm{M}}_{n}$ is a composition of two linear order isomorphisms, and is therefore a linear order isomorphism itself. Due to Proposition 4 , considering any $\tilde{f}$ in $\mathcal{L}\left(\mathcal{X}^{n} \times \mathcal{R}\right) / I_{\mathcal{P}_{n}}, \mathrm{M}_{n}$ is constant on $\tilde{f}$, and the value it takes on any element of $\tilde{f}$ is exactly $\tilde{\mathbf{M}}_{n}(\tilde{f})$.

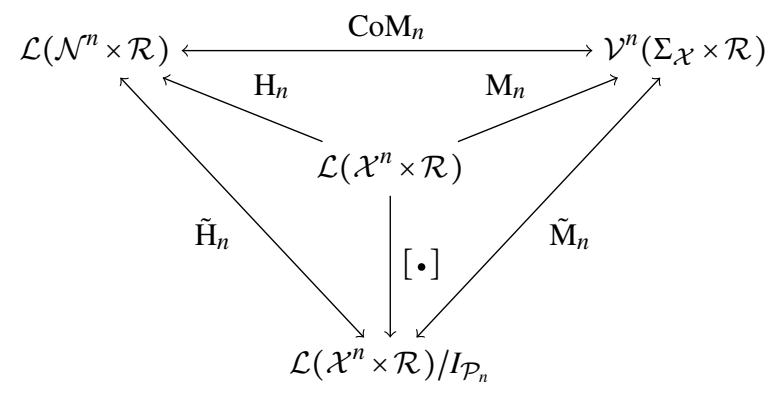

FIGURE 2. Commuting diagram for $\mathrm{CoM}_{n}, \tilde{\mathrm{M}}_{n}$ and $\tilde{\mathrm{H}}_{n}$

The commuting diagram in Figure 2 illustrates the surjections [ $\bullet], \mathrm{H}_{n}$ and $\mathrm{M}_{n}$, and the bijections $\tilde{\mathrm{H}}_{n}, \tilde{\mathrm{M}}_{n}$ and $\operatorname{CoM}_{n}$. It shows that both $\mathcal{L}\left(\mathcal{N}^{n} \times \mathcal{R}\right)$ and $\mathcal{V}^{n}\left(\Sigma_{\mathcal{X}} \times \mathcal{R}\right)$ are orderisomorphic to $\mathcal{L}\left(\mathcal{X}^{n} \times \mathcal{R}\right) / I_{\mathcal{P}_{n}}$, so they are both suitable for defining a representing choice function on: in Theorem 7 we used the space $\mathcal{L}\left(\mathcal{N}^{n} \times \mathcal{R}\right)$, and here, in Theorem 12, we will use the other equivalent space $\mathcal{V}^{n}\left(\Sigma_{\mathcal{X}} \times \mathcal{R}\right)$.

Theorem 12 (Finite polynomial representation). Consider any choice function $C_{n}$ on $\mathcal{L}\left(\mathcal{X}^{n} \times \mathcal{R}\right)$. Then $C_{n}$ is exchangeable if and only if there is a unique representing choice function $\tilde{C}$ on $\mathcal{V}^{n}\left(\Sigma_{\mathcal{X}} \times \mathcal{R}\right)$ such that

$$
C_{n}(A)=\left\{f \in A: \mathrm{M}_{n}(f) \in \tilde{C}\left(\mathrm{M}_{n}(A)\right)\right\} \text { for all } A \text { in } \mathcal{Q}\left(\mathcal{L}\left(\mathcal{X}^{n} \times \mathcal{R}\right)\right) \text {. }
$$


Furthermore, $\tilde{C}$ is then given by $\tilde{C}\left(\mathrm{M}_{n}(A)\right)=\mathrm{M}_{n}\left(C_{n}(A)\right)$ for all $A$ in $\mathcal{Q}\left(\mathcal{L}\left(\mathcal{X}^{n} \times \mathcal{R}\right)\right)$. Finally, $C_{n}$ is coherent if and only if $\tilde{C}$ is. We call $\tilde{C}$ the frequency representation of $C_{n}$.

Similarly, consider any set of desirable gambles $D_{n} \subseteq \mathcal{L}\left(\mathcal{X}^{n} \times \mathcal{R}\right)$. Then $D_{n}$ is exchangeable if and only if there is a unique representing set of desirable gambles $\tilde{D} \subseteq \mathcal{V}^{n}\left(\Sigma_{\mathcal{X}} \times \mathcal{R}\right)$ such that $D_{n}=\bigcup \tilde{\mathrm{M}}_{n}^{-1}(\tilde{D})$. Furthermore, in that case, $\tilde{D}$ is given by $\tilde{D}=\mathrm{M}_{n}\left(D_{n}\right)$. Finally, $D_{n}$ is coherent if and only if $\tilde{D}$ is. We call $\tilde{D}$ the frequency representation of $D_{n}$.

Proof. The part for desirability has essentially already been proved in Reference [6, Theorem 21]. Here, we give a shorter alternative proof that also works for choice functions.

Let $C^{\prime \prime}$ on $\mathcal{L}\left(\mathcal{N}^{n} \times \mathcal{R}\right)$ and $D^{\prime \prime} \subseteq \mathcal{L}\left(\mathcal{N}^{n} \times \mathcal{R}\right)$ be the representing choice function and set of desirable gambles from Theorem 7 and let $\tilde{C}$ be defined by

$$
\operatorname{CoM}_{n}(f) \in \tilde{C}\left(\operatorname{CoM}_{n}(A)\right) \Leftrightarrow f \in C^{\prime \prime}(A)
$$

for all $A$ in $\mathcal{Q}\left(\mathcal{L}\left(\mathcal{N}^{n} \times \mathcal{R}\right)\right)$ and $f$ in $A$, and $\tilde{D}:=\operatorname{CoM}_{n}\left(D^{\prime \prime}\right)$. Since $\operatorname{CoM}_{n}$ is a linear order isomorphism, $\tilde{C}$ and $\tilde{D}$ are unique, and $\mathrm{M}_{n}(f) \in \tilde{C}\left(\mathrm{M}_{n}(A)\right) \Leftrightarrow \mathrm{H}_{n}(f) \in C^{\prime \prime}\left(\mathrm{H}_{n}(A)\right)$ for all $A$ in $\mathcal{Q}\left(\mathcal{L}\left(\mathcal{X}^{n}\right) \times \mathcal{R}\right)$ and $f$ in $A$, and $\tilde{D}=\operatorname{CoM}_{n}\left(\mathrm{H}_{n}\left(D_{n}\right)\right)=\mathrm{M}_{n}\left(D_{n}\right)$, and all the coherence properties are preserved, from which the statements follow.

4.6. Conditioning in terms of polynomials. It turns out that conditioning an exchangeable choice function can be done very easily using the frequency representation. Assume that we observe a count vector $\check{m}$ in $\mathcal{N}^{\check{n}}$, and we condition the choice function $C$ on [ $\check{m}$ ], to obtain the conditional choice function $\left.C\rfloor \check{m}:=\operatorname{marg}_{\hat{n}}(C][\check{m}]\right)$ on $\mathcal{L}\left(\mathcal{X}^{\hat{n}} \times \mathcal{R}\right)$, which is exchangeable by Propositions 8 and 9 What is its frequency representation, then?

Proposition 13. Consider any coherent and exchangeable choice function $C$ on $\mathcal{L}\left(\mathcal{X}^{n} \times \mathcal{R}\right)$, and any $\check{m}$ in $\mathcal{N}^{n}$. If $\tilde{C}$ on $\mathcal{V}^{n}\left(\Sigma_{\mathcal{X}} \times \mathcal{R}\right)$ is the frequency representation of $C$, then the frequency representation of $C\rfloor \breve{m}$ is the choice function $\tilde{C}\rfloor \breve{m}$ on $\mathcal{V}^{\hat{n}}\left(\Sigma_{\mathcal{X}} \times \mathcal{R}\right)$, defined by

$$
\hat{h} \in \tilde{C}\rfloor \check{m}(\hat{A}) \Leftrightarrow B_{\check{m}} \hat{h} \in \tilde{C}\left(B_{\check{m}} \hat{A}\right) \text {, for all } \hat{A} \text { in } \mathcal{Q}\left(\mathcal{V}^{\hat{n}}\left(\Sigma_{\mathcal{X}} \times \mathcal{R}\right)\right) \text { and } \hat{h} \text { in } \hat{A}
$$

Proof. Since $C$ is exchangeable with frequency representation $\tilde{C}$, by Theorem 12 we have that

$$
C(A)=\left\{f \in A: \mathrm{M}_{n}(f) \in \tilde{C}\left(\mathrm{M}_{n}(A)\right)\right\} \text { for all } A \text { in } \mathcal{Q}\left(\mathcal{L}\left(\mathcal{X}^{n} \times \mathcal{R}\right)\right) .
$$

Consider any $\check{x}$ in $[\check{m}]$. Then $C] \breve{m}=C] \check{x}$ by Proposition 9, so

$$
C] \breve{m}(\hat{A})=C \mid \check{x}(\hat{A})=\left\{\hat{f} \in \hat{A}: \mathrm{M}_{n}\left(\mathbb{I}_{\{\check{x}\}} \hat{f}\right) \in \tilde{C}\left(\mathrm{M}_{n}\left(\mathbb{I}_{\{\check{x}\}} \hat{A}\right)\right)\right\} \text { for all } \hat{A} \text { in } \mathcal{Q}\left(\mathcal{L}\left(\mathcal{X}^{\hat{n}} \times \mathcal{R}\right)\right) \text {. }
$$

It suffices to show that $\mathrm{M}_{n}\left(\mathbb{I}_{\{\check{x}\}} \hat{f}\right)=\frac{1}{|[\check{m}]|} B_{\check{m}} \mathrm{M}_{\hat{n}}(\hat{f})$ and $\mathrm{M}_{n}\left(\mathbb{I}_{\{\check{x}\}} \hat{A}\right)=\frac{1}{|[\check{m}]|} B_{\check{m}} \mathrm{M}_{\hat{n}}(\hat{A})$, since then indeed

$$
\begin{aligned}
C] \breve{m}(\hat{A}) & =\left\{\hat{f} \in \hat{A}: \mathrm{M}_{n}\left(\mathbb{I}_{\{\check{x}\}} \hat{f}\right) \in \tilde{C}\left(\mathrm{M}_{n}\left(\mathbb{I}_{\{\check{x}\}} \hat{A}\right)\right)\right\} \\
& =\left\{\hat{f} \in \hat{A}:|[\check{m}]| \mathrm{M}_{n}\left(\mathbb{I}_{\{\check{x}\}} \hat{f}\right) \in \tilde{C}\left(|[\check{m}]| \mathrm{M}_{n}\left(\mathbb{I}_{\{\check{x}\}} \hat{A}\right)\right)\right\} \\
& =\left\{\hat{f} \in \hat{A}: B_{\breve{m}} \mathrm{M}_{\hat{n}}(\hat{f}) \in \tilde{C}\left(B_{\check{m}} \mathrm{M}_{\hat{n}}(\hat{A})\right)\right\} \\
& \left.=\left\{\hat{f} \in \hat{A}: \mathrm{M}_{\hat{n}}(\hat{f}) \in \tilde{C}\right] \check{m}\left(\mathrm{M}_{\hat{n}}(\hat{A})\right)\right\} \text { for all } \hat{A} \text { in } \mathcal{Q}\left(\mathcal{L}\left(\mathcal{X}^{\hat{n}} \times \mathcal{R}\right)\right),
\end{aligned}
$$

taking coherence of $\tilde{C}$ [more specifically, Axiom $\mathrm{C4a}$ into account, so $\tilde{C}] \breve{m}$ is the frequency representation of $C] \breve{m}$. Since $\mathrm{M}_{n}$ works element-wise on $\mathbb{I}_{\{\breve{x}\}} \hat{A}$, it even suffices to show that $\mathrm{M}_{n}\left(\mathbb{I}_{\{\check{x}\}} \hat{f}\right)=\frac{1}{|[\check{m}]|} B_{\check{m}} \mathrm{M}_{\hat{n}}(\hat{f})$ for every $\hat{f}$ in $\hat{A}$. Lemma 14 establishes this.

Lemma 14. Consider any $\check{n}<n, \check{m}$ in $\mathcal{N}^{\check{n}}$, $\check{x}$ in $\check{m}$ and $\hat{f}$ in $\mathcal{L}\left(\mathcal{X}^{\hat{n}} \times \mathcal{R}\right)$. Then $\mathrm{M}_{n}\left(\mathbb{I}_{\{\check{x}\}} \hat{f}\right)=$ $\frac{1}{[[\check{m}] \mid} B_{\check{m}} \mathrm{M}_{\hat{n}}(\hat{f})$ 
Proof. Consider any $\hat{f}$ in $\hat{A}$, any $\theta$ in $\Sigma_{\mathcal{X}}$ and any $r$ in $\mathcal{R}$. Then

$$
\mathrm{M}_{n}\left(\mathbb{I}_{\{\check{x}\}} \hat{f}\right)(\theta, r)=\sum_{m \in \mathcal{N}^{n}} \sum_{y \in[m]} \mathbb{I}_{\{\check{x}\}} \hat{f}(y, r) \prod_{z \in \mathcal{X}} \theta_{z}^{m_{z}} .
$$

Consider some $m$ in $\mathcal{N}$. Since

$$
\mathbb{I}_{\{\check{x}\}} \hat{f}(y, r)=\left\{\begin{array}{ll}
\hat{f}\left(y_{\check{n}+1}, \ldots, y_{n}, r\right) & \text { if }\left(y_{1}, \ldots, x_{\check{n}}\right)=\check{x} \\
0 & \text { if }\left(y_{1}, \ldots, x_{\check{n}}\right) \neq \check{x}
\end{array} \text { for all } y=\left(y_{1}, \ldots, y_{n}\right) \text { in }[m]\right.
$$

we have that $m \geq \check{m} \Leftrightarrow \sum_{y \in[m]} \mathbb{I}_{\{\breve{x}\}} \hat{f}(y, r) \neq 0$, so

$$
\mathrm{M}_{n}\left(\mathbb{I}_{\{\check{x}\}} \hat{f}\right)(\theta, r)=\sum_{\substack{m \in \mathcal{N}^{n} \\ m \geq \check{m}}} \sum_{y \in[m]} \mathbb{I}_{\{\check{x}\}} \hat{f}(y, r) \prod_{z \in \mathcal{X}} \theta_{z}^{m_{z}}
$$

Consider some $m$ in $\mathcal{N}^{n}$ Then $m \geq \check{m}$ if and only if $\hat{m}:=m-\check{m} \geq 0$, so $m \geq \check{m}$ if and only if $\hat{m} \in \mathcal{N}^{\hat{n}}$. In that case, $\hat{m}$ is a count vector itself. Assume that $m \geq \check{m}$. Then

$$
\begin{aligned}
\sum_{y \in[m]} \mathbb{I}_{\{\breve{x}\}} \hat{f}(y, r) & =\sum_{\left(y_{1}, \ldots, y_{n}\right) \in[m]} \mathbb{I}_{\{\breve{x}\}} \hat{f}\left(y_{1}, \ldots, y_{n}, r\right) \\
& =\sum_{\left(y_{1}, \ldots, y_{\breve{n}}\right)=\check{x}\left(y_{\check{n}+1}, \ldots, y_{n}\right) \in[\hat{m}]} \mathbb{I}_{\{\check{x}\}} \hat{f}\left(y_{1}, \ldots, y_{n}, r\right) \\
& =\sum_{\left(y_{\check{n}+1}, \ldots, y_{n}\right) \in[\hat{m}]} \hat{f}\left(y_{\check{n}+1}, \ldots, y_{n}, r\right)=\sum_{y \in[\hat{m}]} \hat{f}(y, r),
\end{aligned}
$$

where the third equality follows from Equation (11). Furthermore,

$$
\prod_{z \in \mathcal{X}} \theta_{z}^{m_{z}}=\prod_{z \in \mathcal{X}} \theta_{z}^{\check{m}_{z}+\hat{m}_{z}}=\prod_{z \in \mathcal{X}} \theta_{z}^{\check{m}_{z}} \prod_{z \in \mathcal{X}} \theta_{z}^{\hat{m}_{z}}=\frac{1}{|[\check{m}]|} B_{\breve{m}}(\theta) \prod_{z \in \mathcal{X}} \theta_{z}^{\hat{m}_{z}} .
$$

Taking these observations into account, we find that

$$
\mathrm{M}_{n}\left(\mathbb{I}_{\{\check{x}\}} \hat{f}\right)(\theta, r)=\frac{1}{|[\check{m}]|} B_{\check{m}}(\theta) \sum_{\hat{m} \in \mathcal{N}^{\hat{n}}} \sum_{y \in[\hat{m}]} \hat{f}(y, r) \prod_{z \in \mathcal{X}} \theta_{z}^{\hat{m}_{z}}=\frac{1}{|[\check{m}]|} B_{\check{m}}(\theta) \mathrm{M}_{\hat{n}}(\hat{f})(\theta, r) .
$$

Since our choice of $\theta$ in $\Sigma_{\mathcal{X}}$ and $r$ in $\mathcal{R}$ was arbitrary, therefore indeed $\mathrm{M}_{n}\left(\mathbb{I}_{\{\check{x}\}} \hat{f}\right)=$ $\frac{1}{|[\check{m}]|} B_{\breve{m}} \mathrm{M}_{\hat{n}}(\hat{f})$.

\section{Countable exchangeability}

In the previous section, we assumed a finite sequence $X_{1}, \ldots, X_{n}$ to be exchangeable, and inferred representation theorems. Here, we will consider the countable sequence $X_{1}, \ldots, X_{n}$, ... to be exchangeable, and derive representation theorems for such an assessment. We will call $\mathcal{X}^{\mathbb{N}}:=\times_{j \in \mathbb{N}} \mathcal{X}$, the set of all possible countable sequences where each variable takes values in $\mathcal{X}$.

First, we need a way to relate gambles on different domains. This will be done using cylindrical extension:

$$
f^{*}(x, r):=f\left(x_{1}, \ldots, x_{n}, r\right) \text { for all } x:=\left(x_{1}, \ldots, x_{n}, \ldots\right) \text { in } \mathcal{X}^{\mathbb{N}} \text { and } r \text { in } \mathcal{R} .
$$

for any gamble $f$ on $\mathcal{X}^{n} \times \mathcal{R}$.

Formally, $f^{*}$ belongs to $\mathcal{L}\left(\mathcal{X}^{\mathbb{N}} \times \mathcal{R}\right)$ while $f$ belongs to $\mathcal{L}\left(\mathcal{X}^{n} \times \mathcal{R}\right)$. However, they contain the same information, and therefore, are indistinguishable from a behavioural point of view. We will resort to the simplifying device of identifying $f$ with its cylindrical extension $f^{*}$. 
Next, we need a way to relate choice functions and sets of desirable gambles on different domains. We will do this using marginalisation: Given any choice function $C$ on $\mathcal{L}\left(\mathcal{X}^{\mathbb{N}} \times \mathcal{R}\right)$ and any $n$ in $\mathbb{N}$, its $\mathcal{X}^{n}$-marginal $C_{n}$ is determined by

$$
C_{n}(A):=C(A) \text { for all } A \text { in } \mathcal{Q}\left(\mathcal{L}\left(\mathcal{X}^{n}\right) \times \mathcal{R}\right) \text {. }
$$

Similarly, given any set of desirable gambles $D \subseteq \mathcal{L}\left(\mathcal{X}^{\mathbb{N}} \times \mathcal{R}\right)$ and any $n$ in $\mathbb{N}$, its $\mathcal{X}^{n}$ marginal $D_{n}$ is $D_{n}:=D \cap \mathcal{L}\left(\mathcal{X}^{n} \times \mathcal{R}\right)$.

Coherence is preserved under marginalisation [it is an immediate consequence of the definition; see, amongst others, Reference [5, Proposition 6] for sets of desirable gambles].

Proposition 15. Consider any coherent choice function $C$ on $\mathcal{L}\left(\mathcal{X}^{\mathbb{N}} \times \mathcal{R}\right)$ and any coherent set of desirable gambles $D \subseteq \mathcal{L}\left(\mathcal{X}^{\mathbb{N}} \times \mathcal{R}\right)$. Then for every $n$ in $\mathbb{N}$, their $\mathcal{X}^{n}$-marginals $C_{n}$ and $D_{n}$ are coherent.

5.1. Vector-valued gambles of finite structure. Before we can explain what it means to assess a countable sequence to be exchangeable, we need to realise that now there are infinitely many variables. From an operational point of view, it will be impossible to describe choice between gambles that depend upon an infinite number of variables. Indeed, since we can never observe the actual outcome in a finite time, gambles will never be actually paid off, and hence every assessment is essentially without any risk. But, it does make operational and behavioural sense to consider choices between gambles of finite structure: gambles that each depend on a finite number of variables only. See Reference [4, Section 3.2] for a discussion.

Definition 8 (Gambles of finite structure). We will call any vector-valued gamble that depends only on a finite number of variables a vector-valued gamble of finite structure. We collect all such gambles in the set $\overline{\mathcal{L}}\left(\mathcal{X}^{\mathbb{N}} \times \mathcal{R}\right)$ :

$$
\overline{\mathcal{L}}\left(\mathcal{X}^{\mathbb{N}} \times \mathcal{R}\right):=\left\{f \in \mathcal{L}\left(\mathcal{X}^{\mathbb{N}} \times \mathcal{R}\right):(\exists n \in \mathbb{N}) f \in \mathcal{L}\left(\mathcal{X}^{n} \times \mathcal{R}\right)\right\}=\bigcup_{n \in \mathbb{N}} \mathcal{L}\left(\mathcal{X}^{n} \times \mathcal{R}\right)
$$

$\overline{\mathcal{L}}\left(\mathcal{X}^{\mathbb{N}} \times \mathcal{R}\right)$ is a linear space, with the usual ordering $\leq$ : for any $f$ and $g$ in $\overline{\mathcal{L}}\left(\mathcal{X}^{\mathbb{N}} \times \mathcal{R}\right)$, $f \leq g \Leftrightarrow f(x, r) \leq g(x, r)$ for all $x$ in $\mathcal{X}^{\mathbb{N}}$ and $r$ in $\mathcal{R}$.

Due to our finitary approach, we can even establish a converse result to Proposition 15 . whose proof is a straightforward verification of all the axioms.

Proposition 16. Consider any choice function $C$ on $\overline{\mathcal{L}}\left(\mathcal{X}^{\mathbb{N}} \times \mathcal{R}\right)$, and any set of desirable gambles $D \subseteq \overline{\mathcal{L}}\left(\mathcal{X}^{\mathbb{N}} \times \mathcal{R}\right)$. If for every $n$ in $\mathbb{N}$, its $\mathcal{X}^{n}$-marginal $C_{n}$ on $\mathcal{L}\left(\mathcal{X}^{n} \times \mathcal{R}\right)$ is coherent, then $C$ is coherent. Similarly, if for every $n$ in $\mathbb{N}$, its $\mathcal{X}^{n}$-marginal $D_{n} \subseteq \mathcal{L}\left(\mathcal{X}^{n} \times \mathcal{R}\right)$ is coherent, then $D$ is coherent.

Proof. We restrict ourselves to proving this for choice functions; the proof for desirability can be found in Reference [4, Proposition 4]. Consider any $A$ in $\mathcal{Q}\left(\overline{\mathcal{L}}\left(\mathcal{X}^{\mathbb{N}} \times \mathcal{R}\right)\right)$. Then any $f$ in $A$ depends - besides on the value of $\mathcal{R}$ - on a finite number of variables $n_{f} \in \mathbb{N}$. Let $n:=\max \left\{n_{f}: f \in A\right\}$, which is a well-defined natural number since $A$ is finite. Every (cylindrical extension of) $f$ in $A$ is then a gamble on $\mathcal{X}^{n}$. It follows then from Equation (12) that $C(A)=C_{n}(A)$.

The proof follows readily once we realise that, following the procedure above, for every option set $A$ there is some $n$ in $\mathbb{N}$ such that $C(A)=C_{n}(A)$; for Axiom C3, we need to consider $A \cup A_{1} \cup A_{2}$ rather than $A$. 
5.2. Set of indifferent gambles. If a subject assesses the sequence of variables $X_{1}, \ldots$, $X_{n}, \ldots$ to be exchangeable, this means that he is indifferent between any gamble $f$ in $\overline{\mathcal{L}}\left(\mathcal{X}^{\mathbb{N}} \times \mathcal{R}\right)$ and its permuted variant $\pi^{t} f$, for any $\pi$ in $\mathcal{P}_{n}$, where $n$ now is the (finite) number of variables that $f$ depends upon: his set of indifferent gambles is

$$
I_{\mathcal{P}}:=\left\{f \in \overline{\mathcal{L}}\left(\mathcal{X}^{\mathbb{N}} \times \mathcal{R}\right):(\exists n \in \mathbb{N}) f \in I_{\mathcal{P}_{n}}\right\}=\bigcup_{n \in \mathbb{N}} I_{\mathcal{P}_{n}} .
$$

If we want to use $I_{\mathcal{P}}$ to define countable exchangeability, it must be a coherent set of indifferent gambles.

Proposition 17. The set $I_{\mathcal{P}}$ is a coherent set of indifferent gambles.

Proof. For Axiom [1], since, by Proposition 3, $0 \in I_{\mathcal{P}_{n}}$ for every $n$ in $\mathbb{N}$, also $0 \in I_{\mathcal{P}}$. For Axiom I2 consider any $f$ in $I_{\mathcal{P}}$, then there is some $n$ in $\mathbb{N}$ for which $f \in I_{\mathcal{P}_{n}}$. By Proposition 3 , we infer that indeed $f \nless 0$ and $f \ngtr 0$. For Axioms 13 and 14 , consider any $f_{1}, f_{2}$ and $f_{3}$ in $I_{\mathcal{P}}$ and any $\lambda$ in $\mathbb{R}$. Then there are $n_{i}$ in $\mathbb{N}$ such that $f_{i} \in I_{\mathcal{P}_{n_{i}}}$, for every $i$ in $\{1,2,3\}$. Let $n:=\max \left\{n_{1}, n_{2}, n_{3}\right\}$. Then $f_{1}, f_{2}$ and $f_{3}$ are elements of $I_{\mathcal{P}_{n}}$, so $\lambda f_{1} \in I_{\mathcal{P}_{n}}$ and $f_{2}+f_{3} \in I_{\mathcal{P}_{n}}$ by Proposition 3 Then indeed $\lambda f_{1} \in I_{\mathcal{P}}$ and $f_{2}+f_{3} \in I_{\mathcal{P}}$, so $I_{\mathcal{P}}$ is indeed a linear space.

Countable exchangeability is now easily defined, similarly to the finitary definition.

Definition 9. A choice function $C$ on $\overline{\mathcal{L}}\left(\mathcal{X}^{\mathbb{N}} \times \mathcal{R}\right)$ is called (countably) exchangeable if $C$ is compatible with $I_{\mathcal{P}}$. Similarly, a set of desirable gambles $D \subseteq \overline{\mathcal{L}}\left(\mathcal{X}^{\mathbb{N}} \times \mathcal{R}\right)$ is called (countably) exchangeable if it is compatible with $I_{\mathcal{P}}$.

This definition is closely related to its finitary counterpart:

Proposition 18. Consider any coherent choice function $C$ on $\overline{\mathcal{L}}\left(\mathcal{X}^{\mathbb{N}} \times \mathcal{R}\right)$. Then $C$ is exchangeable if and only if for every $n$ in $\mathbb{N}$, the $\mathcal{X}^{n}$-marginal $C_{n}$ of $C$ is exchangeable. Similarly, consider any coherent set of desirable gambles $D \subseteq \overline{\mathcal{L}}\left(\mathcal{X}^{\mathbb{N}} \times \mathcal{R}\right)$. Then $D$ is exchangeable if and only if for every choice of $n$ in $\mathbb{N}$, the $\mathcal{X}^{n}$-marginal $D_{n}$ of $D$ is exchangeable.

Proof. The proof for sets of desirable real-valued gambles, in the more general context of partial exchangeability, can be found in Reference [4, Proposition 18], and can be trivially extended to vector-valued gambles.

We give the proof for choice functions. For necessity, assume that $C$ is exchangeable, or equivalently, that $C$ is compatible with $I_{\mathcal{P}}$. Use Equation (1) to infer that then, equivalently,

$$
\left(\forall \tilde{h} \in I_{\mathcal{P}}\right)\left(\forall \tilde{A} \in \mathcal{Q}\left(\overline{\mathcal{L}}\left(\mathcal{X}^{\mathbb{N}} \times \mathcal{R}\right)\right)\right)(\{0, \tilde{h}\} \subseteq \tilde{A} \Rightarrow(0 \in C(\tilde{A}) \Leftrightarrow \tilde{h} \in C(\tilde{A}))) .
$$

Consider any $n$ in $\mathbb{N}$. We need to prove that then $C_{n}$ is compatible with $I_{\mathcal{P}_{n}}$, or equivalently, that

$$
\left(\forall h \in I_{\mathcal{P}_{n}}\right)\left(\forall A \in \mathcal{Q}\left(\mathcal{L}\left(\mathcal{X}^{n} \times \mathcal{R}\right)\right)\right)\left(\{0, h\} \subseteq A \Rightarrow\left(0 \in C_{n}(A) \Leftrightarrow h \in C_{n}(A)\right)\right) .
$$

So consider any $A \supseteq\{0\}$ in $\mathcal{Q}\left(\mathcal{L}\left(\mathcal{X}^{n} \times \mathcal{R}\right)\right)$ and $h$ in $A$. Then $A$ is an element of $\mathcal{Q}\left(\overline{\mathcal{L}}\left(\mathcal{X}^{\mathbb{N}} \times\right.\right.$ $\mathcal{R}))$ and $h$ an element of $\overline{\mathcal{L}}\left(\mathcal{X}^{\mathbb{N}} \times \mathcal{R}\right)$, so $0 \in C(A) \Leftrightarrow h \in C(A)$ by Equation (13). Therefore, after marginalising, $0 \in C_{n}(A) \Leftrightarrow h \in C_{n}(A)$, so $C_{n}$ is compatible with $I_{\mathcal{P}_{n}}$, and by Definition 9 therefore indeed exchangeable.

For sufficiency, assume that $C_{n}$ is exchangeable for every $n$ in $\mathbb{N}$-so it satisfies Equation (14) for every $n$ in $\mathbb{N}$. We need to prove that then $C$ is exchangeable. Using Equation (13), it suffices to consider any $\tilde{A}$ in $\mathcal{Q}\left(\overline{\mathcal{L}}\left(\mathcal{X}^{\mathbb{N}} \times \mathcal{R}\right)\right)$ such that $0 \in \tilde{A}$, and any $\tilde{h}$ in $\tilde{A}$, and prove that $0 \in C(\tilde{A}) \Leftrightarrow \tilde{h} \in C(\tilde{A})$. Since $\tilde{A} \cup\{\tilde{h}\}$ consists of gambles of finite structure, there is some (sufficiently large) $n$ in $\mathbb{N}$ for which $\tilde{A} \in \mathcal{Q}\left(\mathcal{L}\left(\mathcal{X}^{n} \times \mathcal{R}\right)\right)$, and therefore also $\tilde{h} \in \mathcal{L}\left(\mathcal{X}^{n} \times \mathcal{R}\right)$. 
Then by Equation (14), $0 \in C_{n}(\tilde{A}) \Leftrightarrow \tilde{h} \in C_{n}(\tilde{A})$, so $0 \in C(\tilde{A}) \Leftrightarrow \tilde{h} \in C(\tilde{A})$, whence $C$ is compatible with $I_{\mathcal{P}}$, and therefore indeed exchangeable.

5.3. A representation theorem for countable sequences. We will look for a similar representation result to the finite case. However, since we no longer deal with finite sequences of length $n$, now the representing choice function will no longer be defined on $\mathcal{V}^{n}\left(\Sigma_{\mathcal{X}} \times \mathcal{R}\right)$, but instead on $\mathcal{V}\left(\Sigma_{\mathcal{X}} \times \mathcal{R}\right)$.

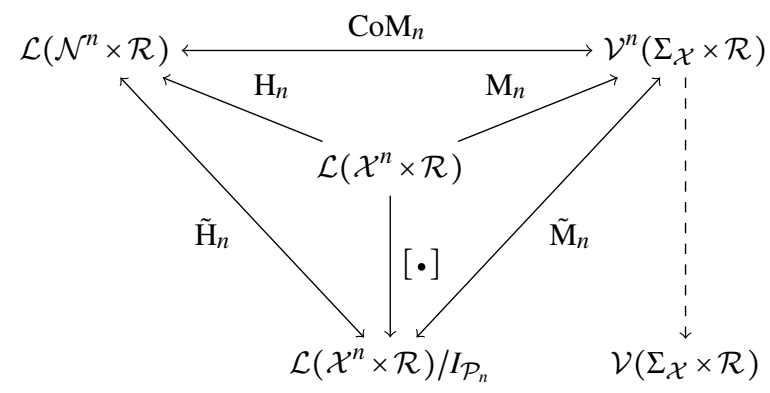

FIGURE 3. Commuting diagram for countable exchangeability

Consider the commuting diagram of Figure 3 , where a dashed line represents an embedding: for every $n$ in $\mathbb{N}, \mathcal{V}^{n}\left(\Sigma_{\mathcal{X}} \times \mathcal{R}\right)$ is a subspace of $\mathcal{V}\left(\Sigma_{\mathcal{X}} \times \mathcal{R}\right)$. This shows the importance of the polynomial representation.

As we have seen, in order to define coherent choice functions on some linear space, we need to provide it with a vector ordering. Similar to what we did before, we use the proper cone $\{0\} \cup \operatorname{posi}\left(\left\{B_{m}: m \in \mathcal{N}^{n}, n \in \mathbb{N}\right\}\right)$ to define the order $\leq_{B}$ on $\mathcal{V}\left(\Sigma_{\mathcal{X}} \times \mathcal{R}\right)$ :

$$
h_{1} \leq_{B} h_{2} \Leftrightarrow(\forall r \in \mathcal{R}) h_{2}(\bullet, r)-h_{1}(\bullet, r) \in\{0\} \cup \operatorname{posi}\left(\left\{B_{m}: m \in \mathcal{N}^{n}, n \in \mathbb{N}\right\}\right)
$$

for all $h_{1}$ and $h_{2}$ in $\mathcal{V}\left(\Sigma_{\mathcal{X}} \times \mathcal{R}\right)$.

Keeping Propositions 15 and 16 in mind, the following results are not surprising.

Proposition 19. Consider any choice function $C^{\prime}$ on $\mathcal{V}\left(\Sigma_{\mathcal{X}} \times \mathcal{R}\right)$. Then $C^{\prime}$ is coherent if and only if for every $n$ in $\mathbb{N}$ the choice function $C_{n}^{\prime}$, defined by $C_{n}^{\prime}(A):=C^{\prime}(A)$ for all $A$ in $\mathcal{Q}\left(\mathcal{V}^{n}\left(\Sigma_{\mathcal{X}} \times \mathcal{R}\right)\right)$, is coherent.

Proof. We only prove sufficiency, since necessity is trivial. So consider any $C^{\prime}$ on $\mathcal{V}\left(\Sigma_{\mathcal{X}} \times\right.$ $\mathcal{R}$ ) such that for every $n$ in $\mathbb{N}, C_{n}^{\prime}$ is coherent. We prove that then $C^{\prime}$ is coherent.

For Axiom $\mathrm{C1}$, consider any $A$ in $\mathcal{Q}\left(\mathcal{V}\left(\Sigma_{\mathcal{X}} \times \mathcal{R}\right)\right)$. Then every polynomial in the finite set $A$ has a certain degree; let $n$ be the maximum of those degrees. Then $A \in \mathcal{Q}\left(\mathcal{V}^{n}\left(\Sigma_{\mathcal{X}} \times \mathcal{R}\right)\right)$, whence indeed $C^{\prime}(A)=C_{n}^{\prime}(A) \neq \varnothing$, since $C_{n}^{\prime}$ is coherent.

For Axiom C2 consider any $h_{1}$ and $h_{2}$ in $\mathcal{V}\left(\Sigma_{\mathcal{X}} \times \mathcal{R}\right)$ such that $h_{1}<_{B} h_{2}$. Then $h_{2}(\cdot, r)-$ $h_{1}(\cdot, r) \in\{0\} \cup \operatorname{posi}\left(\left\{B_{m}: m \in \mathcal{N}^{n}, n \in \mathbb{N}\right\}\right)$ for all $r$ in $\mathcal{R}$, and $h_{2}(\cdot, r)-h_{1}(\bullet, r) \in \operatorname{posi}\left(\left\{B_{m}:\right.\right.$ $\left.\left.m \in \mathcal{N}^{n}, n \in \mathbb{N}\right\}\right)$ for some $r$ in $\mathcal{R}$. Let $\mathcal{R}^{\prime}:=\left\{r \in \mathcal{R}: h_{2}(\cdot, r)-h_{1}(\bullet, r) \neq 0\right\} \subseteq \mathcal{R}$ be the nonempty set of rewards for which $h_{2}(\bullet, r)-h_{1}(\bullet, r)$ belongs to posi $\left(\left\{B_{m}: m \in \mathcal{N}^{n}, n \in \mathbb{N}\right\}\right)$; then $h_{2}(\bullet, r)-h_{1}(\cdot, r)=0$ for all $r$ in $\mathcal{R} \backslash \mathcal{R}^{\prime}$. Consider, for every $r$ in $\mathcal{R}^{\prime}$, a representing polynomial $p_{r}$ of $h_{1}(\bullet, r)$, and let $n_{1}$ be the degree of the representing polynomial in the finite set $\left\{p_{r}: r \in \mathcal{R}^{\prime}\right\}$ with highest degree. Then, for every $r$ in $\mathcal{R}^{\prime}, h_{1}(\cdot, r)$ is represented by a polynomial in $\mathcal{V}^{n_{1}}\left(\Sigma_{\mathcal{X}}\right)$. Similarly, we find that, for every $r$ in $\mathcal{R}^{\prime}, h_{2}(\bullet, r)$ is represented by a polynomial in $\mathcal{V}^{n_{2}}\left(\Sigma_{\mathcal{X}}\right)$ for some $n_{2}$ in $\mathbb{N}$. Let $n:=\max \left\{n_{1}, n_{2}\right\}$. Then, for every $r$ 
in $\mathcal{R}^{\prime}$, there is a representing polynomial of $h_{2}(\bullet, r)-h_{1}(\bullet, r)$ whose degree is not higher than $n$, so $h_{2}-h_{1} \in \mathcal{V}^{n}\left(\Sigma_{\mathcal{X}}\right)$ and therefore $h_{2}(\bullet, r)-h_{1}(\bullet, r) \in \operatorname{posi}\left(\left\{B_{m}: m \in \mathcal{N}^{n}\right\}\right)$. Since $h_{2}(\bullet, r)-h_{1}(\bullet, r)=0$ for every $r$ in $\mathcal{R} \backslash \mathcal{R}^{\prime}$, this guarantees that $(\forall r \in \mathcal{R}) h_{2}(\bullet, r)-h_{1}(\bullet, r) \epsilon$ $\{0\} \cup \operatorname{posi}\left(\left\{B_{m}: m \in \mathcal{N}^{n}, n \in \mathbb{N}\right\}\right)$, or, in other words, that $h_{1}<_{B}^{n} h_{2}$, whence indeed $h_{1} \notin$ $C_{n}^{\prime}\left(\left\{h_{1}, h_{2}\right\}\right)=C^{\prime}\left(\left\{h_{1}, h_{2}\right\}\right)$, since $C_{n}^{\prime}$ is coherent.

For Axiom $\mathrm{C} 3$, consider any $A, A_{1}$ and $A_{2}$ in $\mathcal{V}\left(\Sigma_{\mathcal{X}} \times \mathcal{R}\right)$. Using the similar construction as for Axiom C2 2 we find that then $A \cup A_{1} \cup A_{2} \subseteq \mathcal{V}^{n}\left(\Sigma_{\mathcal{X}} \times \mathcal{R}\right)$, for some $n$ in $\mathbb{N}$. Then $A, A_{1}$ and $A_{2}$ all are elements of $\mathcal{Q}\left(\mathcal{V}^{n}\left(\Sigma_{\mathcal{X}} \times \mathcal{R}\right)\right)$. For Axiom C3a. assume that $C^{\prime}\left(A_{2}\right) \subseteq A_{2} \backslash A_{1}$ and $A_{1} \subseteq A_{2} \subseteq A$. Then $C_{n}^{\prime}\left(A_{2}\right) \subseteq A_{2} \backslash A_{1}$ and therefore, since $C_{n}^{\prime}$ is coherent, indeed $C^{\prime}(A)=$ $C_{n}^{\prime}(A) \subseteq A \backslash A_{1}$. For Axiom C3b assume that $C^{\prime}\left(A_{2}\right) \subseteq A_{2} \backslash A_{1}$ and $A \subseteq A_{1}$. Then $C_{n}^{\prime}\left(A_{2}\right) \subseteq$ $A_{2} \backslash A_{1}$ and therefore, since $C_{n}^{\prime}$ is coherent, indeed $C^{\prime}\left(A_{2} \backslash A\right)=C_{n}^{\prime}\left(A_{2} \backslash A\right) \subseteq A_{2} \backslash A_{1}$.

For Axiom C4, consider any $h$ in $\mathcal{V}\left(\Sigma_{\mathcal{X}} \times \mathcal{R}\right)$, any $\lambda$ in $\mathbb{R}_{>0}$ and any $A_{1}$ and $A_{2}$ in $\mathcal{Q}\left(\mathcal{V}\left(\Sigma_{\mathcal{X}} \times \mathcal{R}\right)\right)$. Using the similar construction as for Axiom C2 , we find that then $\{h\} \cup$ $A_{1} \cup A_{2} \subseteq \mathcal{V}^{n}\left(\Sigma_{\mathcal{X}} \times \mathcal{R}\right)$, for some $n$ in $\mathbb{N}$. Then $h \in \mathcal{V}^{n}\left(\Sigma_{\mathcal{X}} \times \mathcal{R}\right)$ and $A_{1}$ and $A_{2}$ both are elements of $\mathcal{Q}\left(\mathcal{V}^{n}\left(\Sigma_{\mathcal{X}} \times \mathcal{R}\right)\right)$. Assume that $A_{1} \subseteq C^{\prime}\left(A_{2}\right)=C_{n}^{\prime}\left(A_{2}\right)$, then, since $C_{n}^{\prime}$ is coherent, indeed $\lambda A_{1} \subseteq C_{n}^{\prime}\left(\lambda A_{2}\right)=C^{\prime}\left(\lambda A_{2}\right)$ and $A_{1}+\{h\} \subseteq C_{n}^{\prime}\left(A_{2}+\{h\}\right)=C^{\prime}\left(A_{2}+\{h\}\right)$.

Theorem 20 (Countable representation). Consider any choice function $C$ on $\overline{\mathcal{L}}\left(\mathcal{X}^{\mathbb{N}} \times \mathcal{R}\right)$. Then $C$ is coherent and exchangeable if and only if there is a coherent representing choice function $\tilde{C}$ on $\mathcal{V}\left(\Sigma_{\mathcal{X}} \times \mathcal{R}\right)$ such that, for every $n$ in $\mathbb{N}$, the $\mathcal{X}^{n}$-marginal $C_{n}$ of $C$ is determined by

$$
C_{n}(A)=\left\{f \in A: \mathrm{M}_{n}(f) \in \tilde{C}\left(\mathrm{M}_{n}(A)\right)\right\} \text { for all } A \text { in } \mathcal{Q}\left(\mathcal{L}\left(\mathcal{X}^{n} \times \mathcal{R}\right)\right) .
$$

In that case, $\tilde{C}$ is uniquely determined via its corresponding rejection function $\tilde{R}$ by $\tilde{R}(\tilde{A}):=$ $\bigcup_{n \in \mathbb{N}} \tilde{R}_{n}\left(\tilde{A} \cap \mathcal{V}^{n}\left(\Sigma_{\mathcal{X}} \times \mathcal{R}\right)\right)$ for all $\tilde{A}$ in $\mathcal{Q}\left(\mathcal{V}\left(\Sigma_{\mathcal{X}} \times \mathcal{R}\right)\right)$, with $\tilde{R}_{n}\left(\mathrm{M}_{n}(A)\right):=\mathrm{M}_{n}\left(R_{n}(A)\right)$ for every $A$ in $\mathcal{Q}\left(\mathcal{L}\left(\mathcal{X}^{n} \times \mathcal{R}\right)\right)$, and where we let $\tilde{R}_{n}(\varnothing):=\varnothing$ for notational convenience. We call $\tilde{C}$ the frequency representation of $C$.

Similarly, consider any set of desirable gambles $D \subseteq \overline{\mathcal{L}}\left(\mathcal{X}^{\mathbb{N}} \times \mathcal{R}\right)$. Then $D$ is coherent and exchangeable if and only if there is a unique representing $\tilde{D} \subseteq \mathcal{V}\left(\Sigma_{\mathcal{X}} \times \mathcal{R}\right)$ such that, for every $n$ in $\mathbb{N}$, the $\mathcal{X}^{n}$-marginal $D_{n}$ is given by $D_{n}=\cup \tilde{\mathrm{M}}_{n}^{-1}\left(\tilde{D} \cap \mathcal{V}^{n}\left(\Sigma_{\mathcal{X}} \times \mathcal{R}\right)\right)$. In that case, $\tilde{D}$ is given by $\tilde{D}=\bigcup_{n \in \mathbb{N}} \mathrm{M}_{n}\left(D_{n}\right)$.

Proof. We begin with the representation of choice functions. That $C$ is exchangeable is, by Proposition 18, equivalent to $C_{n}$ is exchangeable, for every $n$ in $\mathbb{N}$. Therefore, by Theorem 12 this is equivalent to:

$$
(\forall n \in \mathbb{N}) C_{n}(A)=\left\{f \in A: \mathrm{M}_{n}(f) \in \tilde{C}_{n}\left(\mathrm{M}_{n}(A)\right)\right\} \text { for all } A \text { in } \mathcal{Q}\left(\mathcal{L}\left(\mathcal{X}^{n} \times \mathcal{R}\right)\right),
$$

where, as a consequence, for every $n$ in $\mathbb{N}, \tilde{C}_{n}$ is uniquely given by

$$
\tilde{C}_{n}\left(\mathrm{M}_{n}(A)\right)=\mathrm{M}_{n}\left(C_{n}(A)\right) \text { for all } A \text { in } \mathcal{Q}\left(\mathcal{L}\left(\mathcal{X}^{n} \times \mathcal{R}\right)\right) \text {. }
$$

That $C$ is coherent, is by Propositions 15 and 16 equivalent to $C_{n}$ is coherent, for every $n$ in $\mathbb{N}$, and using Theorem 12 , to $\tilde{C}_{n}$ is coherent, for every $n$ in $\mathbb{N}$. We prove that this is necessary and sufficient for the existence of some coherent choice function $\tilde{C}$ on $\mathcal{V}\left(\Sigma_{\mathcal{X}} \times \mathcal{R}\right)$ that, for every $n$ in $\mathbb{N}$, satisfies Equation 15 .

We start by showing the converse implication (sufficiency). Assume that there is some coherent choice function $\tilde{C}$ on $\mathcal{V}\left(\Sigma_{\mathcal{X}} \times \mathcal{R}\right)$ that for every $n$ in $\mathbb{N}$ satisfies Equation (15). Consider any $n$ in $\mathbb{N}$. By Proposition 19 , then the choice function $\tilde{C}_{n}$ on $\mathcal{V}^{n}\left(\Sigma_{\mathcal{X}} \times \mathcal{R}\right)$, determined by $\tilde{C}_{n}\left(\tilde{A}_{n}\right)=\tilde{C}\left(\tilde{A}_{n}\right)$ for every $A_{n}$ in $\mathcal{Q}\left(\mathcal{V}^{n}\left(\Sigma_{\mathcal{X}} \times \mathcal{R}\right)\right)$, is coherent. Furthermore, by Equation (15, it satisfies indeed

$$
C_{n}(A)=\left\{f \in A: \mathrm{M}_{n}(f) \in \tilde{C}_{n}\left(\mathrm{M}_{n}(A)\right)\right\} \text { for all } A \text { in } \mathcal{Q}\left(\mathcal{L}\left(\mathcal{X}^{n} \times \mathcal{R}\right)\right) .
$$


Since $\tilde{C}_{n}\left(\tilde{A}_{n}\right)=\tilde{C}\left(\tilde{A}_{n}\right)$ for every $\tilde{A}_{n}$ in $\mathcal{Q}\left(\mathcal{V}^{n}\left(\Sigma_{\mathcal{X}} \times \mathcal{R}\right)\right)$, we find that also

$$
\tilde{R}\left(\tilde{A}_{n}\right)=\tilde{R}_{n}\left(\tilde{A}_{n}\right) \text { for all } \tilde{A}_{n} \text { in } \mathcal{Q}\left(\mathcal{V}^{n}\left(\Sigma_{\mathcal{X}} \times \mathcal{R}\right)\right) .
$$

Next, we show that then $\tilde{R}(\tilde{A})=\bigcup_{n \in \mathbb{N}} \tilde{R}_{n}\left(\tilde{A} \cap \mathcal{V}^{n}\left(\Sigma_{\mathcal{X}} \times \mathcal{R}\right)\right)$ for all $\tilde{A}$ in $\mathcal{Q}\left(\mathcal{V}\left(\Sigma_{\mathcal{X}} \times \mathcal{R}\right)\right)$, thus making it unique. Consider any $\tilde{A}$ in $\mathcal{Q}\left(\mathcal{V}\left(\Sigma_{\mathcal{X}} \times \mathcal{R}\right)\right)$. Then, by the definition of a polynomial gamble, there is some $n^{*}$ in $\mathbb{N}$ such that $\tilde{A} \in \mathcal{Q}\left(\mathcal{V}^{n^{*}}\left(\Sigma_{\mathcal{X}} \times \mathcal{R}\right)\right)$, whence $\tilde{A}=\tilde{A} \cap \mathcal{V}^{n^{*}}\left(\Sigma_{\mathcal{X}} \times \mathcal{R}\right)$ and therefore $\tilde{R}(\tilde{A})=\tilde{R}\left(\tilde{A} \cap \mathcal{V}^{n^{*}}\left(\Sigma_{\mathcal{X}} \times \mathcal{R}\right)\right)$. By Equation (16) therefore $\tilde{R}(\tilde{A})=\tilde{R}_{n^{*}}\left(\tilde{A} \cap \mathcal{V}^{n^{*}}\left(\Sigma_{\mathcal{X}} \times \mathcal{R}\right)\right)$, whence indeed $\tilde{R}(\tilde{A}) \subseteq \bigcup_{n \in \mathbb{N}} \tilde{R}_{n}\left(\tilde{A} \cap \mathcal{V}^{n}\left(\Sigma_{\mathcal{X}} \times \mathcal{R}\right)\right)$. Conversely, consider any $h$ in $\bigcup_{n \in \mathbb{N}} \tilde{R}_{n}\left(\tilde{A} \cap \mathcal{V}^{n}\left(\Sigma_{\mathcal{X}} \times \mathcal{R}\right)\right)$. Then $h \in \tilde{R}_{n^{*}}\left(\tilde{A} \cap \mathcal{V}^{n^{*}}\left(\Sigma_{\mathcal{X}} \times \mathcal{R}\right)\right)$ for some $n^{*}$ in $\mathbb{N}$, and therefore, by Equation $\left(16, h \in \tilde{R}\left(\tilde{A} \cap \mathcal{V}^{n^{*}}\left(\Sigma_{\mathcal{X}} \times \mathcal{R}\right)\right)\right.$. By Axiom R3a. therefore indeed $h \in \tilde{R}(\tilde{A})$.

We complete the proof by showing the direct implication (necessity). Assume that

$$
(\forall n \in \mathbb{N}) C_{n}(A)=\left\{f \in A: \mathrm{M}_{n}(f) \in \tilde{C}_{n}\left(\mathrm{M}_{n}(A)\right)\right\} \text { for all } A \text { in } \mathcal{Q}\left(\mathcal{L}\left(\mathcal{X}^{n} \times \mathcal{R}\right)\right),
$$

and that $\tilde{C}_{n}$ is coherent for every $n$ in $\mathbb{N}$. Let the rejection function $\tilde{R}$ on $\mathcal{V}\left(\Sigma_{\mathcal{X}} \times \mathcal{R}\right)$ by determined by $\tilde{R}(\tilde{A}):=\bigcup_{n \in \mathbb{N}} \tilde{R}_{n}\left(\tilde{A} \cap \mathcal{V}^{n}\left(\Sigma_{\mathcal{X}} \times \mathcal{R}\right)\right)$ for all $\tilde{A}$ in $\mathcal{Q}\left(\mathcal{V}\left(\Sigma_{\mathcal{X}} \times \mathcal{R}\right)\right)$. The proof is finished if we can show that it satisfies Equation (16) for every $n$ in $\mathbb{N}$, because, if it does, Equation (15) is then satisfied, and furthermore, by Proposition 19 it is then coherent. Consider any $n^{*}$ in $\mathbb{N}$. We need to show that $\bigcup_{n \in \mathbb{N}} \tilde{R}_{n}\left(\tilde{A}_{n^{*}} \cap \mathcal{V}^{n}\left(\Sigma_{\mathcal{X}} \times \mathcal{R}\right)\right)=$ $\tilde{R}_{n^{*}}\left(\tilde{A}_{n^{*}}\right)$ for all $\tilde{A}_{n^{*}}$ in $\mathcal{Q}\left(\mathcal{V}^{n^{*}}\left(\Sigma_{\mathcal{X}} \times \mathcal{R}\right)\right)$. Observe that $\tilde{A}_{n^{*}} \cap \mathcal{V}^{n}\left(\Sigma_{\mathcal{X}} \times \mathcal{R}\right)=\tilde{A}_{n^{*}}$ for every $n \geq n^{*}$, whence by Lemma 21. $\bigcup_{n \in \mathbb{N}} \tilde{R}_{n}\left(\tilde{A}_{n^{*}} \cap \mathcal{V}^{n}\left(\Sigma_{\mathcal{X}} \times \mathcal{R}\right)\right)=\bigcup_{n \leq n^{*}} \tilde{R}_{n}\left(\tilde{A}_{n^{*}} \cap \mathcal{V}^{n}\left(\Sigma_{\mathcal{X}} \times\right.\right.$ $\mathcal{R})$ ). Furthermore, since $\tilde{A}_{n^{*}} \cap \mathcal{V}^{n_{1}}\left(\Sigma_{\mathcal{X}} \times \mathcal{R}\right) \subseteq \tilde{A}_{n^{*}} \cap \mathcal{V}^{n_{2}}\left(\Sigma_{\mathcal{X}} \times \mathcal{R}\right)$ whenever $n_{1} \leq n_{2}$, also $\tilde{R}_{n_{1}}\left(\tilde{A}_{n^{*}} \cap \mathcal{V}^{n_{1}}\left(\Sigma_{\mathcal{X}} \times \mathcal{R}\right)\right)=\tilde{R}_{n_{2}}\left(\tilde{A}_{n^{*}} \cap \mathcal{V}^{n_{1}}\left(\Sigma_{\mathcal{X}} \times \mathcal{R}\right)\right) \subseteq \tilde{R}_{n_{2}}\left(\tilde{A}_{n^{*}} \cap \mathcal{V}^{n_{2}}\left(\Sigma_{\mathcal{X}} \times \mathcal{R}\right)\right)$, where the first equality follows from Lemma 21, and the second one from Axiom R3a. This implies that indeed $\bigcup_{n \leq n^{*}} \tilde{R}_{n}\left(\tilde{A}_{n^{*}} \cap \mathcal{V}^{n}\left(\Sigma_{\mathcal{X}} \times \mathcal{R}\right)\right)=\tilde{R}_{n^{*}}\left(\tilde{A}_{n^{*}} \cap \mathcal{V}^{n^{*}}\left(\Sigma_{\mathcal{X}} \times \mathcal{R}\right)\right)=\tilde{R}_{n^{*}}\left(\tilde{A}_{n^{*}}\right)$.

The representation of sets of desirable gambles is a trivial extension to vector-valued gambles of the proof given in Reference [4, Theorem 22].

Lemma 21. Consider any coherent choice function $C$ on $\overline{\mathcal{L}}\left(\mathcal{X}^{\mathbb{N}} \times \mathcal{R}\right)$ and assume that, for every $n$ in $\mathbb{N}$, its $\mathcal{X}^{n}$-marginal $C_{n}$ is given by

$$
C_{n}(A)=\left\{f \in A: \mathrm{M}_{n}(f) \in \tilde{C}_{n}\left(\mathrm{M}_{n}(A)\right)\right\} \text { for all } A \text { in } \mathcal{Q}\left(\mathcal{L}\left(\mathcal{X}^{n} \times \mathcal{R}\right)\right) \text {, }
$$

where $\tilde{C}_{n}$ is a coherent choice function on $\mathcal{V}^{n}\left(\Sigma_{\mathcal{X}} \times \mathcal{R}\right)$. Then, for every $n_{1} \leq n_{2}$ in $\mathbb{N}$ and $\tilde{A}_{n_{1}}$ in $\mathcal{V}^{n_{1}}\left(\Sigma_{\mathcal{X}} \times \mathcal{R}\right)$ :

$$
\tilde{C}_{n_{1}}\left(\tilde{A}_{n_{1}}\right)=\tilde{C}_{n_{2}}\left(\tilde{A}_{n_{1}}\right) \text {. }
$$

Proof. Since $\mathrm{M}_{n_{1}}$ is surjective, we can find $A_{n_{1}}$ in $\mathcal{Q}\left(\mathcal{L}\left(\mathcal{X}^{n_{1}} \times \mathcal{R}\right)\right)$ such that $\mathrm{M}_{n_{1}}\left(A_{n_{1}}\right)=\tilde{A}_{n_{1}}$. We will show that $\tilde{R}_{n_{1}}\left(\tilde{A}_{n_{1}}\right)=\tilde{R}_{n_{2}}\left(\tilde{A}_{n_{1}}\right)$.

To show that $\tilde{R}_{n_{1}}\left(\tilde{A}_{n_{1}}\right) \subseteq \tilde{R}_{n_{2}}\left(\tilde{A}_{n_{1}}\right)$, consider any $h$ in $\tilde{R}_{n_{1}}\left(\tilde{A}_{n_{1}}\right)$, and let $f_{n_{1}}$ be an element of $A_{n_{1}}$ such that $\mathrm{M}_{n_{1}}\left(f_{n_{1}}\right)=h$. Then $f_{n_{1}} \in R_{n_{1}}\left(A_{n_{1}}\right)$ by Equation (17). If we denote the cylindrical extension of $A_{n_{1}}$ to $\mathcal{X}^{n_{2}}$ by $A_{n_{2}}$ - and the cylindrical extension of $f_{n_{1}}$ by $f_{n_{2}}$ 一, then $f_{n_{2}} \in R_{n_{2}}\left(A_{n_{2}}\right)$ because $R_{n_{1}}$ and $R_{n_{2}}$ are related through marginalisation. But $R_{n_{2}}\left(A_{n_{2}}\right)=$ $\left\{f \in A_{n_{2}}: \mathrm{M}_{n_{2}}(f) \in \tilde{R}_{n_{2}}\left(\mathrm{M}_{n_{2}}\left(A_{n_{2}}\right)\right)\right\}$ by Equation (17), and by Lemma 22, $R_{n_{2}}\left(A_{n_{2}}\right)=\{f \epsilon$ $\left.A_{n_{2}}: \mathrm{M}_{n_{2}}(f) \in \tilde{R}_{n_{2}}\left(\mathrm{M}_{n_{1}}\left(A_{n_{1}}\right)\right)\right\}$. Since $f_{n_{2}} \in R_{n_{2}}\left(A_{n_{2}}\right)$, indeed $h=\mathrm{M}_{n_{1}}\left(f_{n_{1}}\right)=\mathbf{M}_{n_{2}}\left(f_{n_{2}}\right) \epsilon$ $\tilde{R}_{n_{2}}\left(\mathrm{M}_{n_{1}}\left(A_{n_{1}}\right)\right)=\tilde{R}_{n_{2}}\left(\tilde{A}_{n_{1}}\right)$.

The proof that also $\tilde{R}_{n_{2}}\left(\tilde{A}_{n_{1}}\right) \subseteq \tilde{R}_{n_{1}}\left(\tilde{A}_{n_{1}}\right)$ is completely similar [with the same notation, $h \in \tilde{R}_{n_{2}}\left(\tilde{A}_{n_{1}}\right)$ implies that $f_{n_{1}} \in R_{n_{2}}\left(A_{n_{1}}\right)$ by Equation [17), which implies that $f_{n_{1}} \in R_{n_{1}}\left(A_{n_{1}}\right)$ because $R_{n_{1}}$ and $R_{n_{2}}$ are related through marginalisation, which in turn implies that $h \in$ $\left.\tilde{R}_{n_{1}}\left(\tilde{A}_{n_{1}}\right)\right]$. 
Lemma 22 ([4, Lemma 27]). Consider any $n_{1} \leq n_{2}$ in $\mathbb{N}$, and any gamble $f_{n_{1}}$ in $\mathcal{L}\left(\mathcal{X}^{n_{1}} \times \mathcal{R}\right)$. Denote its cylindrical extension to $\mathcal{X}^{n_{2}}$ by $f_{n_{2}}$. Then $\mathrm{M}_{n_{1}}\left(f_{n_{1}}\right)=\mathrm{M}_{n_{2}}\left(f_{n_{2}}\right)$.

5.4. Conditioning and countable representation. We use the same notation and ideas as in Reference [6, Section 5.2] for desirability, and generalise it to choice models.

Suppose we have a coherent and exchangeable choice function $C$ on $\overline{\mathcal{L}}\left(\mathcal{X}^{\mathbb{N}} \times \mathcal{R}\right)$ with associated frequency representation $\tilde{C}$ on $\mathcal{V}\left(\Sigma_{\mathcal{X}} \times \mathcal{R}\right)$. Suppose that we observe the values $\check{x}$ of the first $\check{n}$ variables, with associated count vector $\check{m}:=T(\check{x})$. We have seen in Proposition 18 that, for every $n$ in $\mathbb{N}$, the $\mathcal{X}^{n}$-marginal $C_{n}$ is exchangeable, and in Proposition 8 that $\left.C_{n}\right\rfloor \check{m}$ is exchangeable (if $n>\check{m}$ ). But what about $\left.C\right\rfloor \check{m}$ ?

Theorem 23. Consider any coherent and exchangeable choice function $C$ on $\overline{\mathcal{L}}\left(\mathcal{X}^{\mathbb{N}} \times \mathcal{R}\right)$ with associated frequency representation $\tilde{C}$ on $\mathcal{V}\left(\Sigma_{\mathcal{X}} \times \mathcal{R}\right)$. After conditioning on a sample with count vector $\check{m}$ in $\left.\mathcal{N}^{\check{n}}, C\right\rfloor \check{m}$ is still exchangeable and coherent, and has frequency representation $\tilde{C} \mid \check{m}$, defined by

$$
h \in \tilde{C}\rfloor \breve{m}(\tilde{A}) \Leftrightarrow B_{\breve{m}} h \in \tilde{C}\left(B_{\breve{m}} \tilde{A}\right) \text {, for every } \tilde{A} \text { in } \mathcal{V}\left(\Sigma_{\mathcal{X}} \times \mathcal{R}\right) \text { and } h \text { in } \tilde{A} \text {. }
$$

Proof. Use Theorem 20 to infer that $\tilde{C}$ is coherent. We first show that $\tilde{C}\rfloor \breve{m}$ is coherent. For Axiom $C 1$. consider any $\tilde{A}$ in $\mathcal{V}\left(\Sigma_{\mathcal{X}} \times \mathcal{R}\right)$. Since $\tilde{C}\left(B_{\check{m}} \tilde{A}\right) \neq \varnothing$, indeed also $\left.\tilde{C}\right] \check{m}(\tilde{A}) \neq \varnothing$.

For Axiom C2, consider any $h_{1}$ and $h_{2}$ in $\mathcal{V}\left(\Sigma_{\mathcal{X}} \times \mathcal{R}\right)$ such that $h_{1}<_{B} h_{2}$-meaning $h_{1} \leq_{B} h_{2}$ and $h_{1} \neq h_{2}$. Then $h_{1}(\bullet, r)-h_{2}(\bullet, r) \in \operatorname{posi}\left(\left\{B_{m}: m \in \mathcal{N}^{n}, n \in \mathbb{N}\right\}\right)$ for some $r$ in $\mathcal{R}$. Let $\mathcal{R}^{\prime}:=\left\{r \in \mathcal{R}: h_{2}(\cdot, r)-h_{1}(\bullet, r) \neq 0\right\} \subseteq \mathcal{R}$ be the non-empty set of rewards for which $h_{2}(\cdot, r)-h_{1}(\cdot, r)$ belongs to posi $\left(\left\{B_{m}: m \in \mathcal{N}^{n}, n \in \mathbb{N}\right\}\right)$. Consider any $r$ in $\mathcal{R}^{\prime}$. Then $h_{1}(\bullet, r)-h_{2}(\bullet, r)=\sum_{i=1}^{\ell} \lambda_{i} B_{m^{i}}$ for some $\ell$ in $\mathbb{N}, \lambda_{1}, \ldots, \lambda_{\ell}$ in $\mathbb{R}_{>0}, n_{1}, \ldots, n_{\ell}$ in $\mathbb{N}$ and $m^{1} \in \mathcal{N}^{n_{1}}, \ldots, m^{\ell} \in \mathcal{N}^{n_{\ell}}$, and therefore $\left(h_{1}(\cdot, r)-h_{2}(\bullet, r)\right) B_{\check{m}}=\sum_{i=1}^{\ell} \lambda_{i} B_{m^{i}} B_{\check{m}}$. Use the result that, for any $\theta$ in $\Sigma_{\mathcal{X}}$,

$$
\begin{aligned}
B_{m^{i}+\check{m}}(\theta)=\left|\left[m^{i}+\check{m}\right]\right| \prod_{z \in \mathcal{X}} \theta_{z}^{m_{z}^{i}+\check{m}_{z}} & =\left|\left[m^{i}+\check{m}\right]\right|\left(\prod_{z \in \mathcal{X}} \theta_{z}^{m_{z}^{i}}\right)\left(\prod_{z \in \mathcal{X}} \theta_{z}^{\check{m}_{z}}\right) \\
& =\frac{\left|\left[m^{i}+\check{m}\right]\right|}{\left|\left[m^{i}\right]\right|[\check{m}] \mid} B_{m^{i}}(\theta) B_{\check{m}}(\theta),
\end{aligned}
$$

so $B_{m^{i}+\check{m}}=\frac{\left|\left[m^{i}+\check{m}\right]\right|}{\left|\left[m^{i}\right]\right|[\check{m}] \mid} B_{m^{i}} B_{\check{m}}$, to infer that

$$
\left(h_{1}(\cdot, r)-h_{2}(\cdot, r)\right) B_{\check{m}}=\sum_{i=1}^{\ell} \lambda_{i} \frac{\left|\left[m^{i}\right]\right||[\check{m}]|}{\left|\left[m^{i}+\check{m}\right]\right|} B_{m^{i}+\check{m}},
$$

so $B_{\breve{m}} h_{1}(\cdot, r)-B_{\breve{m}} h_{2}(\cdot, r) \in \operatorname{posi}\left(\left\{B_{m}: m \in \mathcal{N}^{n}, n \in \mathbb{N}\right\}\right)$. Since the choice of $r$ in $\mathcal{R}^{\prime}$ was arbitrary, and since $0=h_{1}(\bullet, r)-h_{2}(\bullet, r)=B_{\breve{m}} h_{1}(\bullet, r)-B_{\breve{m}} h_{2}(\bullet, r)$ for al $r$ in $\mathcal{R} \backslash \mathcal{R}^{\prime}$, we have $B_{\check{m}} h_{1}<_{B} B_{\breve{m}} h_{2}$, so $B_{\breve{m}} h_{1} \notin \tilde{C}\left(\left\{B_{\check{m}} h_{1}, B_{\check{m}} h_{2}\right\}\right)$ and therefore indeed $\left.h_{1} \notin \tilde{C}\right\rfloor \breve{m}\left(\left\{h_{1}, h_{2}\right\}\right)$.

For Axiom C3a consider any $\tilde{A}, \tilde{A}_{1}$ and $\tilde{A}_{2}$ in $\mathcal{Q}\left(\mathcal{V}\left(\Sigma_{\mathcal{X}} \times \mathcal{R}\right)\right)$ such that $\left.\tilde{C}\right] \check{m}\left(\tilde{A}_{2}\right) \subseteq \tilde{A}_{2} \backslash$ $\tilde{A}_{1}$ and $\tilde{A}_{1} \subseteq \tilde{A}_{2} \subseteq A$. Then $\tilde{C}\left(B_{\breve{m}} \tilde{A}_{2}\right) \subseteq B_{\breve{m}}\left(\tilde{A}_{2} \backslash \tilde{A}_{1}\right)=B_{\check{m}} \tilde{A}_{2} \backslash B_{\breve{m}} \tilde{A}_{1}$ and $B_{\breve{m}} \tilde{A}_{1} \subseteq B_{\check{m}} \tilde{A}_{2} \subseteq B_{\breve{m}} \tilde{A}$, and therefore, by coherence, $\tilde{C}\left(B_{\check{m}} \tilde{A}\right) \subseteq B_{\check{m}} \tilde{A} \backslash B_{\check{m}} \tilde{A}_{1}$. But then indeed $\left.\tilde{C}\right\rfloor \check{m}(\tilde{A}) \subseteq \tilde{A} \backslash \tilde{A}_{1}$.

For Axiom C3b consider any $\tilde{A}, \tilde{A}_{1}$ and $\tilde{A}_{2}$ in $\mathcal{Q}\left(\mathcal{V}\left(\Sigma_{\mathcal{X}} \times \mathcal{R}\right)\right)$ such that $\left.C\right] \check{m}\left(\tilde{A}_{2}\right) \subseteq$ $\tilde{A}_{2} \backslash \tilde{A}_{1}$ and $\tilde{A} \subseteq A_{1}$. Then $C\left(B_{\check{m}} \tilde{A}_{2}\right) \subseteq B_{\check{m}}\left(\tilde{A}_{2} \backslash \tilde{A}_{1}\right)=B_{\breve{m}} \tilde{A}_{2} \backslash B_{\check{m}} \tilde{A}_{1}$ and $B_{\check{m}} \tilde{A} \subseteq B_{\check{m}} \tilde{A}_{1}$, and therefore, $C\left(B_{\check{m}}\left(\tilde{A}_{2} \backslash \tilde{A}\right)\right)=C\left(B_{\check{m}} \tilde{A}_{2} \backslash B_{\check{m}} \tilde{A}\right) \subseteq B_{\check{m}} \tilde{A}_{2} \backslash B_{\check{m}} \tilde{A}_{1}=B_{\check{m}}\left(\tilde{A}_{2} \backslash \tilde{A}_{1}\right)$. But then indeed $C] \breve{m}\left(\tilde{A}_{2} \backslash \tilde{A}\right) \subseteq \tilde{A}_{2} \backslash \tilde{A}_{1}$.

For Axiom C4a consider any $\tilde{A}_{1}$ and $\tilde{A}_{2}$ in $\mathcal{Q}\left(\mathcal{V}\left(\Sigma_{\mathcal{X}} \times \mathcal{R}\right)\right)$ and any $\lambda$ in $\mathbb{R}_{>0}$ for which $\tilde{A}_{1} \subseteq C \mid \check{m}\left(\tilde{A}_{2}\right)$. Then $B_{\check{m}} \tilde{A}_{1} \subseteq C\left(B_{\breve{m}} \tilde{A}_{2}\right)$, and therefore, $B_{\breve{m}} \lambda \tilde{A}_{1} \subseteq C\left(B_{\breve{m}} \lambda \tilde{A}_{2}\right)$. But then indeed $\left.\lambda \tilde{A}_{1} \subseteq C\right] \check{m}\left(\lambda \tilde{A}_{2}\right)$. 
For Axiom C4b consider any $\tilde{A}_{1}$ and $\tilde{A}_{2}$ in $\mathcal{Q}\left(\mathcal{V}\left(\Sigma_{\mathcal{X}} \times \mathcal{R}\right)\right)$ and any $h$ in $\mathcal{V}\left(\Sigma_{\mathcal{X}} \times \mathcal{R}\right)$ for which $\left.\tilde{A}_{1} \subseteq C\right] \breve{m}\left(\tilde{A}_{2}\right)$. Then $B_{\breve{m}} \tilde{A}_{1} \subseteq C\left(B_{\check{m}} \tilde{A}_{2}\right)$, and therefore, $B_{\breve{m}}\left(\tilde{A}_{1}+\{h\}\right)=B_{\breve{m}} \tilde{A}_{1}+$ $\left\{B_{\check{m}} h\right\} \subseteq C\left(B_{\check{m}} \tilde{A}_{2}+\left\{B_{\check{m}} h\right\}\right)=C\left(B_{\check{m}}\left(\tilde{A}_{2}+\{h\}\right)\right)$. But then indeed $\left.\tilde{A}_{1}+\{h\} \subseteq C\right\rfloor \breve{m}\left(\tilde{A}_{2}+\{h\}\right)$.

To finish the proof, it suffices to show that $\tilde{C}] \check{m}$ is the frequency representation of $C\rfloor \breve{m}$. To establish this, we will show that, for every $n$ in $\mathbb{N}$ such that $n>\check{n}$, the $\mathcal{X}^{n}$-marginal $\left.C_{n}\right] \check{m}$ of $C$ conditional on $\check{m}$, is given by

$$
\left.\left.C_{n}\right] \breve{m}(\hat{A})=\left\{f \in \hat{A}: \mathrm{M}_{\hat{n}}(f) \in \tilde{C}\right] \breve{m}\left(\mathrm{M}_{\hat{n}}(\hat{A})\right)\right\} \text { for all } \hat{A} \text { in } \mathcal{Q}\left(\mathcal{L}\left(\mathcal{X}^{\hat{n}} \times \mathcal{R}\right)\right),
$$

where $\hat{n}:=n-\check{n}>1$.

Consider any $n$ in $\mathbb{N}$ such that $n>\check{n}$. Since $C$ is exchangeable, by Theorem 20, we get

$$
C_{n}(A)=\left\{f \in A: \mathrm{M}_{n}(f) \in \tilde{C}\left(\mathrm{M}_{n}(A)\right)\right\} \text { for all } A \text { in } \mathcal{Q}\left(\mathcal{L}\left(\mathcal{X}^{n} \times \mathcal{R}\right)\right) .
$$

Therefore

$$
\left.C_{n}\right] \check{m}(\hat{A})=\left\{f \in \hat{A}: \mathrm{M}_{n}\left(\mathbb{I}_{[\check{m}]} f\right) \in \tilde{C}\left(\mathrm{M}_{n}\left(\mathbb{I}_{[\check{m}]} \hat{A}\right)\right)\right\} \text { for all } \hat{A} \text { in } \mathcal{Q}\left(\mathcal{L}\left(\mathcal{X}^{\hat{n}} \times \mathcal{R}\right)\right) .
$$

By Lemma 14, we have that then

$$
\left.C_{n}\right\rfloor \check{m}(\hat{A})=\left\{f \in \hat{A}: B_{\check{m}} \mathrm{M}_{\hat{n}}(f) \in \tilde{C}\left(B_{\check{m}} \mathrm{M}_{\hat{n}}(\hat{A})\right)\right\} \text { for all } \hat{A} \text { in } \mathcal{Q}\left(\mathcal{L}\left(\mathcal{X}^{\hat{n}} \times \mathcal{R}\right)\right),
$$

and by Equation [18, therefore indeed

$$
\left.\left.C_{n}\right] \breve{m}(\hat{A})=\left\{f \in \hat{A}: \mathrm{M}_{\hat{n}}(f) \in \tilde{C}\right\rfloor \breve{m}\left(\mathrm{M}_{\hat{n}}(\hat{A})\right)\right\} \text { for all } \hat{A} \text { in } \mathcal{Q}\left(\mathcal{L}\left(\mathcal{X}^{\hat{n}} \times \mathcal{R}\right)\right) \text {. }
$$

\section{CONCLUSION}

We have studied exchangeability and we have found counterparts to de Finetti's finite and countable representation results, in the general setting of choice functions. We have shown that an exchangeability assessment is a particular indifference assessment, where we identified the set of indifferent options. The main idea that made (finite) representation

possible is the linear order isomorphism $\tilde{\mathrm{H}}_{n}^{-1}$ between the quotient space and the set of gambles on count vectors, indicating that (finitely) exchangeable choice functions can be represented by a choice function that essentially represents preferences between gambles on the unknown composition $m$ of an urn with $n$ balls of types $\mathcal{X}$. Alternatively, for the countable case, we have shown that there is a polynomial representation.

In their treatment of exchangeability for lower previsions [7], and for sets of desirable gambles [6], the authors investigated exchangeability as a structural assessment. They found an expression for the least informative coherent belief model-be it a coherent lower previsions or a coherent set of desirable gambles-that is exchangeable and that extends some direct assessments. They called this least informative extension under exchangeability the exchangeable natural extension. It is an important line of future research to find such an extension for choice functions. We have clear ideas on how to find expressions for it, and intend to report on this elsewhere.

\section{ACKNOWLEDGMENTS}

Arthur Van Camp's research was partly funded by Banco Santander via Campus de Excelencia Internacional. Gert de Cooman's research was partly funded through project number 3G012512 of the Research Foundation Flanders (FWO). The authors would like to express their gratitude to Enrique Miranda for his detailed reading and stimulating feedback. The authors would also like to thank the anonymous referees of this paper and a previous conference version [24] for their useful and constructive comments. 


\section{REFERENCES}

[1] Mark A. Aizerman. New problems in the general choice theory. Social Choice and Welfare, 2:235-282, 1985. doi: 10.1007/BF00292690.

[2] Kenneth J. Arrow. Social choice and individual values. Cowles Foundation Monographs Series. Yale University Press, 1951.

[3] Seamus Bradley. How to choose among choice functions. In Thomas Augustin, Serena Doria, Enrique Miranda, and Erik Quaeghebeur, editors, ISIPTA '15: Proceedings of the Ninth International Symposium on Imprecise Probability: Theories and Applications, pages 57-66, Pescara, 2015. SIPTA.

[4] Jasper De Bock, Arthur Van Camp, Márcio Alves Diniz, and Gert de Cooman. Representation theorems for partially exchangeable random variables. Fuzzy Sets and Systems, 284:1-30, 2016. doi: 10.1016/j.fss.2014.10.027.

[5] Gert de Cooman and Enrique Miranda. Irrelevance and independence for sets of desirable gambles. Journal of Artificial Intelligence Research, 45:601-640, 2012. doi: 10.1613/jair.3770.

[6] Gert de Cooman and Erik Quaeghebeur. Exchangeability and sets of desirable gambles. International Journal of Approximate Reasoning, 53(3):363-395, 2012. doi: 10.1016/ j.ijar.2010.12.002. Precisely imprecise: A collection of papers dedicated to Henry E. Kyburg, Jr.

[7] Gert de Cooman, Erik Quaeghebeur, and Enrique Miranda. Exchangeable lower previsions. Bernoulli, 15(3):721-735, 2009. doi: 10.3150/09-BEJ182. URL http: //hdl.handle.net/1854/LU-498518.

[8] Bruno de Finetti. La prévision: ses lois logiques, ses sources subjectives. Annales de l'Institut Henri Poincaré, 7:1-68, 1937. English translation in [13].

[9] Persi Diaconis. Finite forms of de finetti's theorem on exchangeability. Synthese, 36: 271-281, 1977.

[10] Junnan He. A generalized unification theorem for choice theoretic foundations: Avoiding the necessity of pairs and triplets. Economics Discussion Paper 2012-23, Kiel Institute for the World Economy, 2012. URL http://www.economics-ejournal. org/economics/discussionpapers/2012-23.

[11] Norman L. Johnson, Samuel Kotz, and Narayanaswamy Balakrishnan. Discrete Multivariate Distributions. Wiley Series in Probability and Statistics. John Wiley and Sons, New York, 1997.

[12] Joseph B. Kadane, Mark J. Schervish, and Teddy Seidenfeld. A Rubinesque theory of decision. Institute of Mathematical Statistics Lecture Notes-Monograph Series, 45:4555, 2004. doi: 10.1214/lnms/1196285378. URL http://www.jstor.org/stable/4356297.

[13] Henry E. Kyburg Jr. and Howard E. Smokler, editors. Studies in Subjective Probability. Wiley, New York, 1964. Second edition (with new material) 1980.

[14] Erik Quaeghebeur. Desirability. In Thomas Augustin, Frank P. A. Coolen, Gert de Cooman, and Matthias C. M. Troffaes, editors, Introduction to Imprecise Probabilities, chapter 1, pages 1-27. John Wiley \& Sons, 2014. doi: 10.1002/9781118763117.ch1.

[15] Herman Rubin. A weak system of axioms for "rational" behavior and the nonseparability of utility from prior. Statistics \& Risk Modeling, 5(1-2):47-58, 1987. doi: 10.1524/strm.1987.5.12.47.

[16] Thomas Schwartz. Rationality and the myth of the maximum. Noûs, 6(2):97-117, 1972. doi: $10.2307 / 2216143$.

[17] Teddy Seidenfeld. Decision theory without "independence" or without "ordering". Economics and Philosophy, 4:267-290, 1988. doi: 10.1017/S0266267100001085. 
[18] Teddy Seidenfeld, Mark J. Schervish, and Joseph B. Kadane. Decisions without ordering. In W. Sieg, editor, Acting and reflecting, volume 211 of Synthese Library, pages 143-170. Kluwer, Dordrecht, 1990.

[19] Teddy Seidenfeld, Mark J. Schervish, and Joseph B. Kadane. Coherent choice functions under uncertainty. Synthese, 172(1):157-176, 2010. doi: 10.1007/ s11229-009-9470-7.

[20] Amartya Sen. Choice functions and revealed preference. The Review of Economic Studies, 38(3):307-317, July 1971. doi: 10.2307/2296384.

[21] Amartya Sen. Social choice theory: A re-examination. Econometrica, 45:53-89, 1977. doi: 10.2307/1913287.

[22] Hirofumi Uzawa. Note on preference and axioms of choice. Annals of the Institute of Statistical Mathematics, 8:35-40, 1956. doi: 10.1007/BF02863564.

[23] Arthur Van Camp. Choice Functions as a Tool to Model Uncertainty. PhD thesis, Ghent University, 2018.

[24] Arthur Van Camp and Gert de Cooman. Exchangeable choice functions. In Alessandro Antonucci, Giorgio Corani, Inés Couso, and Sébastien Destercke, editors, Proceedings of the Tenth International Symposium on Imprecise Probability: Theories and Applications, volume 62 of Proceedings of Machine Learning Research, pages 346-357. PMLR, 2017. URL http://proceedings.mlr.press/v62/van-camp17a.html.

[25] Arthur Van Camp, Gert de Cooman, Enrique Miranda, and Erik Quaeghebeur. Coherent choice functions, desirability and indifference. Fuzzy sets and systems, 2017. doi: https://doi.org/10.1016/j.fss.2017.05.019. In press.

[26] Arthur Van Camp, Gert de Cooman, and Enrique Miranda. Lexicographic choice functions. International Journal of Approximate Reasoning, 92:97-119, 2018. doi: 10.1016/j.ijar.2017.10.011.

[27] John von Neumann and Oskar Morgenstern. Theory of Games and Economic Behaviour. Princeton University Press, 1944. ISBN 0691041830. Third edition 1953.

[28] Peter Walley. Statistical Reasoning with Imprecise Probabilities. Chapman and Hall, London, 1991.

[29] Peter Walley. Towards a unified theory of imprecise probability. International Journal of Approximate Reasoning, 24(2-3):125-148, 2000. doi: 10.1016/S0888-613X(00) 00031-1.

[30] Marco Zaffalon and Enrique Miranda. Axiomatising incomplete preferences through sets of desirable gambles. Journal of Artificial Intelligence Research, 60:1057-1126, 2017.

GHent University, IDLAB, TEChNOLOGIEPARK-ZwiJnAARde 914, 9052 ZWIJNAARde, BelgiUm

Email address: $\{$ arthur.vancamp, gert. decooman\}@ugent . be 